\title{
MAGNETIC FIELDS IN RELATIVISTIC COLLISIONLESS SHOCKS
}

\author{
Rodolfo Santana ${ }^{1}$, Rodolfo Barniol Duran ${ }^{2}$, and Pawan Kumar ${ }^{1}$ \\ ${ }^{1}$ Department of Astronomy, University of Texas at Austin, Austin, TX 78712, USA; santana@astro.as.utexas.edu, pk@astro.as.utexas.edu \\ ${ }^{2}$ Racah Institute for Physics, The Hebrew University, Jerusalem 91904, Israel; rbarniol@ phys.huji.ac.il \\ Received 2013 September 11; accepted 2014 February 12; published 2014 March 21
}

\begin{abstract}
We present a systematic study on magnetic fields in gamma-ray burst (GRB) external forward shocks (FSs). There are 60 (35) GRBs in our X-ray (optical) sample, mostly from Swift. We use two methods to study $\epsilon_{B}$ (fraction of energy in magnetic field in the FS): (1) for the X-ray sample, we use the constraint that the observed flux at the end of the steep decline is $\geqslant$ X-ray FS flux; (2) for the optical sample, we use the condition that the observed flux arises from the FS (optical sample light curves decline as $\sim t^{-1}$, as expected for the FS). Making a reasonable assumption on $E$ (jet isotropic equivalent kinetic energy), we converted these conditions into an upper limit (measurement) on $\epsilon_{B} n^{2 /(p+1)}$ for our X-ray (optical) sample, where $n$ is the circumburst density and $p$ is the electron index. Taking $n=1 \mathrm{~cm}^{-3}$, the distribution of $\epsilon_{B}$ measurements (upper limits) for our optical (X-ray) sample has a range of $\sim 10^{-8}-10^{-3}\left(\sim 10^{-6}-10^{-3}\right)$ and median of $\sim$ few $\times 10^{-5}\left(\sim\right.$ few $\left.\times 10^{-5}\right)$. To characterize how much amplification is needed, beyond shock compression of a seed magnetic field $\sim 10 \mu \mathrm{G}$, we expressed our results in terms of an amplification factor, AF, which is very weakly dependent on $n$ ( $\mathrm{AF} \propto n^{0.21}$ ). The range of AF measurements (upper limits) for our optical (X-ray) sample is $\sim 1-1000(\sim 10-300)$ with a median of $\sim 50(\sim 50)$. These results suggest that some amplification, in addition to shock compression, is needed to explain the afterglow observations.
\end{abstract}

Key words: gamma-ray burst: general - methods: analytical - radiation mechanisms: non-thermal

Online-only material: color figures

\section{INTRODUCTION}

Gamma-ray bursts (GRBs) are bright explosions occurring at cosmological distances that release gamma rays for a brief time, typically on a timescale of $\sim$ few $\times 10$ s (e.g., Piran 2004; Gehrels et al. 2009; Zhang 2011). This short-lived emission of gamma rays is known as the prompt emission. After the prompt emission, long-lived emission in the X-ray, optical, and radio bands (on timescales of days, months, or even years) is also observed from what is called the "afterglow." Although the mechanism for the prompt emission is currently being debated, the afterglow emission has a well-established model based on external shocks (Rees \& Meszaros 1992; Meszaros \& Rees 1993; Paczynski \& Rhoads 1993). In this framework, a relativistic jet emitted by the central engine interacts with the medium surrounding the GRB progenitor. This interaction produces two shocks; the external-reverse shock and the external-forward shock (Meszaros \& Rees 1997; Sari \& Piran 1999b). The external-reverse shock heats up the jet, while the external-forward shock heats up the medium surrounding the explosion. The external-reverse shock is believed to be short-lived in the optical band (Sari \& Piran 1999a) and might have been observed, perhaps, in a few cases. The long-lived afterglow emission is interpreted as synchrotron radiation from the external-forward shock. This shock is taken to produce a power-law distribution of high-energy electrons and to amplify the preexisting seed magnetic field in the surrounding medium. These high-energy electrons are then accelerated by the amplified magnetic field and emit radiation by the synchrotron process.

One of the open questions in the field of GRB afterglows is, what is the dynamo mechanism amplifying magnetic fields in the collisionless relativistic shocks involved for GRB external shocks? The magnetic field strength downstream of the shock front is expressed in terms of $\epsilon_{B}$, which is defined as the fraction of energy that is in the magnetic field downstream of the shock front. With this definition, the explicit expression for $\epsilon_{B}$ is

$$
\epsilon_{B}=\frac{B^{2}}{32 \pi n m_{p} c^{2} \Gamma^{2}},
$$

where $B$ is the co-moving magnetic field downstream of the shock front, $n$ is the density surrounding the GRB progenitor, $m_{p}$ is the proton mass, $c$ is the speed of light, and $\Gamma$ is the Lorentz factor of the shocked fluid downstream of the shock front (e.g., Sari et al. 1998; Wijers \& Galama 1999; Panaitescu \& Kumar 2000). If shock compression is the only mechanism amplifying the magnetic field downstream of the shock front, then $B$ is given by $B=4 \Gamma B_{0}$ (e.g., Achterberg et al. 2001), where $B_{0}$ is the seed magnetic field in the medium surrounding the GRB progenitor. Using this expression for $B, \epsilon_{B}$ simplifies to $\epsilon_{B}=B_{0}^{2} / 2 \pi n m_{p} c^{2}$. Using the value for the ambient magnetic field of the Milky Way $B_{0} \sim$ few $\mu \mathrm{G}$ and a density for the surrounding medium of $n=1 \mathrm{~cm}^{-3}, \epsilon_{B}$ is expected to be $\sim 10^{-9}$.

Several studies have modeled afterglow data to determine what values of the afterglow parameters best describe the observations (e.g., Wijers \& Galama 1999; Panaitescu \& Kumar 2002; Yost et al. 2003; Panaitescu 2005). The results from previous studies show that $\epsilon_{B}$ ranges from $\epsilon_{B} \sim 10^{-5}-10^{-1}$. These values for $\epsilon_{B}$ are much larger than the $\epsilon_{B} \sim 10^{-9}$ expected from shock compression alone and suggest that some additional amplification is needed to explain the observations. There have been several theoretical and numerical studies that have considered possible mechanisms, operating in the plasma in the medium surrounding the GRB, that can generate extra amplification for the magnetic field. The mechanisms that have been proposed are the two-stream Weibel instability (Weibel 1959; Medvedev \& Loeb 1999; Gruzinov \& Waxman 1999; Silva et al. 2003; Medvedev et al. 2005) and dynamo generated by turbulence (Milosavljević \& Nakar 2006; Milosavljevic et al. 2007; 
Sironi \& Goodman 2007; Goodman \& MacFadyen 2008; Couch et al. 2008; Zhang et al. 2009; Mizuno et al. 2011; Inoue et al. 2011).

Recent results (Kumar \& Barniol Duran 2009, 2010) found surprisingly small values of $\epsilon_{B} \sim 10^{-7}$ for three bright GRBs with Fermi/LAT detections. These values of $\epsilon_{B}$ are $\sim 2$ orders of magnitude smaller than the smallest previously reported $\epsilon_{B}$ value, and they can be explained with the only amplification coming from shock compression of a seed magnetic field of a few $10 \mu \mathrm{G}^{3}$ Although this seed magnetic field is stronger than that of the Milky Way by about a factor $\sim 10$, seed magnetic fields of a few $10 \mu \mathrm{G}$ have been measured before. The seed magnetic fields in the spiral arms of some gas-rich spiral galaxies with high star formation rates have been measured to be 20-30 $\mu \mathrm{G}$ (Beck 2011). Seed magnetic fields as high as $0.5-18 \mathrm{mG}$ were measured in starburst galaxies by measuring the Zeeman splitting of the $\mathrm{OH}$ megamaser emission line at $1667 \mathrm{MHz}$ (Robishaw et al. 2008).

Given this disagreement between the recent and previous results, the question regarding the amplification of magnetic fields in GRB external relativistic collisionless shocks remains unanswered. The first goal of this study is to provide a systematic determination of $\epsilon_{B}$ for a large sample of GRBs by using the same method to determine $\epsilon_{B}$ for each burst in our X-ray or optical sample. This is the first time such a large and systematic study has been carried out for $\epsilon_{B}$. Knowing the value of $\epsilon_{B}$ for large samples will help us determine how much amplification of the magnetic field is needed to explain the afterglow observations. We mostly limit our samples to GRBs detected by the Swift satellite, with measured redshift. In this study, we determine an upper limit on $\epsilon_{B}$ for our X-ray sample and a measurement of $\epsilon_{B}$ for our optical sample. We use a new method to determine an upper limit on $\epsilon_{B}$ with X-ray data, which relies on using the steep decline observed by Swift in many $\mathrm{X}$-ray light curves. We expect that the observed flux at the end of the steep decline (EoSD) is larger than the predicted flux from the external-forward shock. Making reasonable assumptions about the other afterglow parameters, we are able to convert this constraint into an upper limit on $\epsilon_{B}$. To determine a measurement of $\epsilon_{B}$ for our optical sample, we restrict our sample to light curves that show a power-law decline with a temporal decay $\sim 1$ at early times, $\sim 10^{2}-10^{3} \mathrm{~s}$, as expected for external-forward shock emission. We choose this selection criterion so that the optical emission is most likely dominated by external-forward shock emission. Making the same reasonable assumptions for the other afterglow parameters and using the condition that the observed flux from the optical light curve is equal to the externalforward shock flux, we are able to convert this condition into a measurement of $\epsilon_{B}$. We also applied a consistency check for the bursts that are in common to our X-ray and optical samples to make sure our results for $\epsilon_{B}$ are correct. The second goal of this study is to determine how much amplification, in addition to shock compression, is needed to explain the results for the $\epsilon_{B}$ upper limits/measurements. To quantify how much amplification beyond shock compression is required by the

\footnotetext{
3 The values given above of $\epsilon_{B} \sim 10^{-7}$ are under the assumption of $n=1 \mathrm{~cm}^{-3}$. It is important to note that when reaching the conclusion that shock compression provides enough amplification to explain the afterglow data, Kumar \& Barniol Duran $(2009,2010)$ did not assume a value for $n$. Also, the results of small $\epsilon_{B} \sim 10^{-7}$ values do not depend on whether or not the LAT emission is produced by the external-forward shock. These small $\epsilon_{B}$ values were inferred from the late-time X-ray and optical data and from the constraint that the external-forward shock does not produce flux at $150 \mathrm{keV}$ that exceeds the observed prompt emission flux at $50 \mathrm{~s}$.
}

observations, we also express the results we found for the $\epsilon_{B}$ upper limits (measurements) for our X-ray (optical) sample in terms of an amplification factor.

This paper is organized as follows. We begin in Section 2 by presenting a review of the values previous studies have found for the microphysical afterglow parameters $\epsilon_{e}$ (the fraction of energy in electrons in the shocked plasma) and $\epsilon_{B}$. In Section 3 (Section 4), we present the method we use to determine an upper limit (measurement) on $\epsilon_{B}$ and apply it to our X-ray (optical) sample of GRBs. In Section 5, we use the GRBs that are in common to both samples to perform a consistency check. We search for a possible correlation between the kinetic energy of the blast wave and $\epsilon_{B}$ in Section 6. In Section 7, we write our results for $\epsilon_{B}$ for our X-ray and optical samples in terms of an amplification factor, which quantifies how much amplification-beyond shock compression-is required by the observations. Lastly, in Section 8, we discuss our results and present our conclusions. The convention we use for the specific flux $f_{v}$, the flux per unit frequency $v$, is $f_{v} \propto v^{-\beta} t^{-\alpha}$. In this convention, $\beta$ is the spectral decay index and $\alpha$ is the temporal decay index. For a GRB at a given redshift $z$, when calculating the luminosity distance to the GRB, $d_{L}$, we used the cosmological parameters $H_{0}=71 \mathrm{~km} \mathrm{~s}^{-1} \mathrm{Mpc}^{-1}, \Omega_{m}=0.27$, and $\Omega_{\Lambda}=0.73$.

\section{LITERATURE REVIEW OF VALUES OF $\epsilon_{e}$ AND $\epsilon_{B}$}

The flux observed from the external-forward shock depends on six parameters: $E, n, s, \epsilon_{e}, \epsilon_{B}$, and $p . E$ is the isotropic equivalent kinetic energy of the jet, and $n$ is the number density of the surrounding medium. The density is taken to be spherically symmetric and to decrease with $r$ as $n(r) \propto$ $r^{-s}$, where $s$ is a constant determining the density profile of the surrounding medium and $r$ is the distance from the center of the explosion. Two cases are usually considered for the density profile: $s=0$ and $s=2$, which respectively correspond to a constant density medium and a wind medium. The microphysical parameters are $\epsilon_{e}$ and $\epsilon_{B}$, where $\epsilon_{e}\left(\epsilon_{B}\right)$ is the fraction of energy in the electrons (magnetic field) in the shocked fluid. The microphysical parameters are taken to be constant throughout the afterglow emission. A power-law distribution of electrons, $d N_{e} / d \gamma_{e} \propto \gamma_{e}^{-p}$ with $\gamma_{e} \geqslant \gamma_{i}$, where $\gamma_{e}$ is the Lorentz factor of the electrons, $\gamma_{i}$ is the minimum Lorentz factor of the electrons, and $N_{e}$ is the number of electrons, is assumed to be produced when the external-forward shock interacts with the surrounding medium. The power-law index of the electron distribution, $p$, is a constant known as the electron index.

In practice, it is very difficult to determine the values of the six afterglow parameters. The value of $p$ and the density profile of the surrounding medium (whether we have an $s=0$ or $s=2$ medium) can be determined from observations of the afterglow spectral decay and temporal decay of the light curve with the so-called closure relations (e.g., Zhang et al. 2006). The remaining four afterglow parameters are more difficult to determine. What is needed to determine these four parameters is observations of the afterglow emission in the four different spectral regimes of the synchrotron afterglow spectrum (we will discuss the afterglow synchrotron spectrum in more detail in Section 3.4). In practice, most GRBs do not have this wealth of observations. In order to determine these four parameters, previous works either have focused only on determining the afterglow parameters for bursts with high-quality data, spanning 

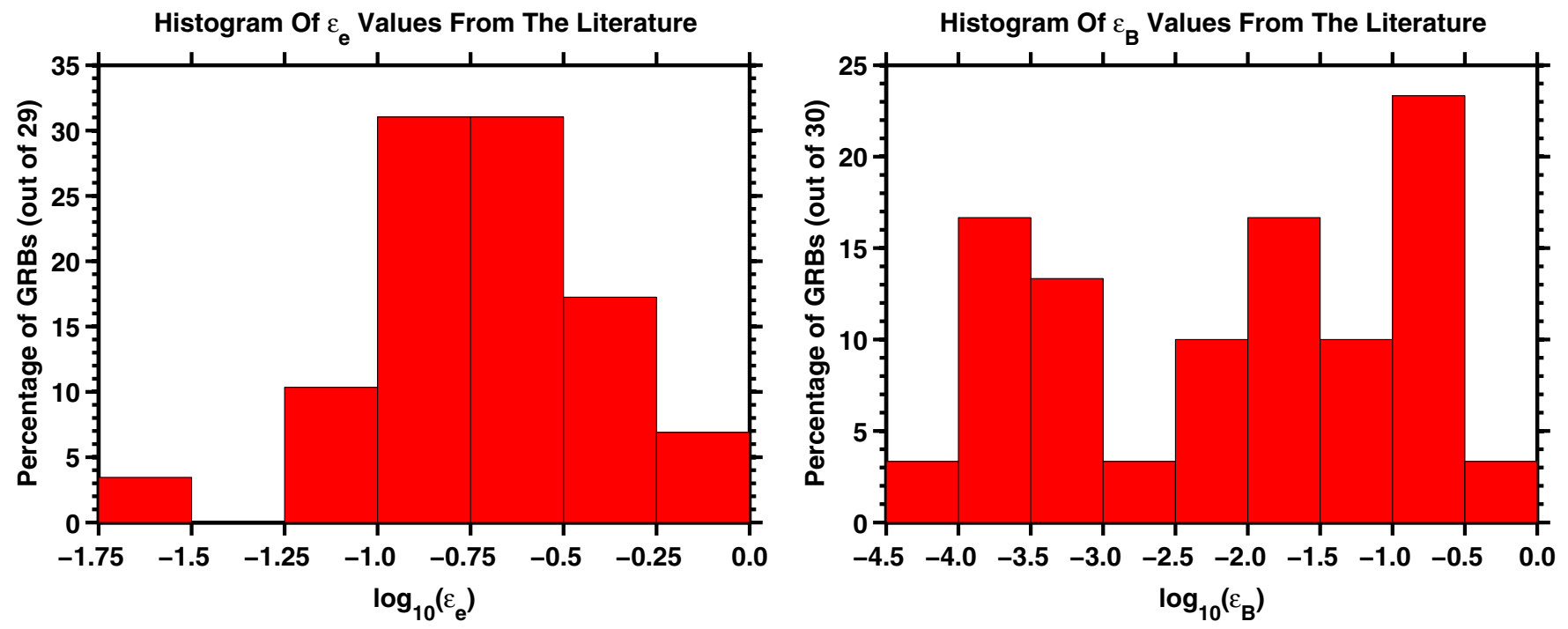

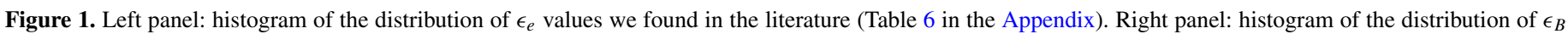
values we found in the literature (Table 6 in Appendix).

(A color version of this figure is available in the online journal.)

all portions of the synchrotron spectrum, or have applied various simplifying assumptions.

We performed a literature search for papers that determine values for $\epsilon_{e}$ and $\epsilon_{B}$ to get an idea of what typical values previous works have found. Different authors applied different techniques for finding $\epsilon_{e}$ and $\epsilon_{B}$. When displaying the results from the literature, we did not discriminate against any method and simply plotted every value we found. However, we did not consider works that made simplifying assumptions when determining $\epsilon_{e}$ and $\epsilon_{B}$, such as equipartition of proton and electron energy $\left(\epsilon_{e}\right)$. The GRBs for which we found $\epsilon_{e}$ and $\epsilon_{B}$ values are shown in Table 6 in the Appendix. Except for GRB 080928, all the GRBs in our sample have radio, optical, and X-ray observations, allowing for a determination of all the afterglow parameters. We included GRB 080928 in our sample because $\epsilon_{e}$ and $\epsilon_{B}$ were able to be uniquely determined from optical and X-ray observations (Rossi et al. 2011).

The $\epsilon_{e}$ values we found in the literature for 29 GRBs are shown in the histogram in the left panel of Figure 1. There is a narrow distribution for $\epsilon_{e}$; it only varies over one order of magnitude, from $\sim 0.02$ to 0.6 , with very few GRBs reported to have $\epsilon_{e}<0.1$. The mean of this distribution is 0.24 , and the median is 0.22 . About $62 \%$ of the GRBs in this sample have $\epsilon_{e} \sim 0.1-0.3$. These results for $\epsilon_{e}$ are also supported by recent simulations of relativistic magnetized collisionless electron-ion shocks presented in Sironi \& Spitkovsky 2011, where they found $\epsilon_{e} \sim 0.2$. The narrow distribution of $\epsilon_{e}$ values from the literature and the results from recent simulations both show that $\epsilon_{e}$ does not change by much from GRB to GRB.

The $\epsilon_{B}$ values we found in the literature for $30 \mathrm{GRBs}$ are shown in the histogram in the right panel of Figure 1. Comparing the two histograms in Figure 1, we can immediately see that there is a much wider range in the distribution of $\epsilon_{B}$, with $\epsilon_{B}$ ranging from $\sim 3.5 \times 10^{-5}$ to 0.33 . A noticeable peak, containing about $24 \%$ of the bursts, is seen in the bin with $-1<$ $\log _{10}\left(\epsilon_{B}\right)<-0.5$. Two other peaks, each containing about $17 \%$ of the GRBs, are seen in the bins with $-2<\log _{10}\left(\epsilon_{B}\right)<-1.5$ and $-4<\log _{10}\left(\epsilon_{B}\right)<-3.5$. The mean of this distribution is $6.3 \times 10^{-2}$, and the median is $1.0 \times 10^{-2}$. The important point of the $\epsilon_{B}$ histogram is that $\epsilon_{B}$ varies over four orders of magnitude, showing that $\epsilon_{B}$ has a wide distribution and is an uncertain parameter.

\section{UPPER LIMIT ON $\epsilon_{B}$ WITH SWIFT X-RAY LIGHT CURVES}

\subsection{Constraining $\epsilon_{B}$ with the X-Ray Light-curve Steep Decline}

One interesting property found by Swift (Gehrels et al. 2004) is that at early times, about $50 \%$ of the light curves detected by the X-ray Telescope (XRT; Burrows et al. 2005) display a very rapid decline in flux, known as the steep decline (Gehrels et al. 2009). The flux during the steep decline typically decays as $t^{-3}$, and it usually lasts $\sim 10^{2}-10^{3} \mathrm{~s}$. By extrapolating the Burst Alert Telescope (BAT; Barthelmy et al. 2005) emission to the X-ray band, O'Brien et al. (2006) showed that there is a continuous transition between the end of the prompt emission and the start of the steep decline phase. This important conclusion led to the interpretation that the X-ray steep decline has an origin associated with the end of the prompt emission.

The favored interpretation for the origin of the steep decline is high-latitude emission (Kumar \& Panaitescu 2000). Although high-latitude emission is able to explain most of the steep decline observations, some GRBs display spectral evolution during the steep decline (Zhang et al. 2007b), which is not expected. In any case, the steep decline cannot be produced by the externalforward shock. Therefore, the observed flux during the steep decline should be larger than or equal to the flux produced by the external-forward shock. We do, however, assume that the time at which the steep decline typically ends, at about $10^{2}-10^{3} \mathrm{~s}$, is past the deceleration time. ${ }^{4}$ For our upper limit on $\epsilon_{B}$ with X-ray data, we will use the expression for the flux from the external-forward shock, which uses the kinetic energy of the blast wave given by the Blandford and McKee solution (Blandford \& McKee 1976). Since this solution is only valid for a decelerating blast wave, we need to be past the deceleration time for it to be applicable.

\footnotetext{
4 The deceleration time marks the time when about half of the kinetic energy of the blast wave has been transferred to the surrounding medium.
} 
Theoretically, it is expected that that the deceleration time occurs before the end of the steep decline. Depending on the density profile of the surrounding medium, the deceleration time $t_{\mathrm{dec}}$ is given by

$$
t_{\mathrm{dec}}= \begin{cases}(220 \mathrm{~s}) E_{53}^{1 / 3} n_{0}^{-1 / 3} \Gamma_{2}^{-8 / 3}(1+z) & s=0 \\ (67 \mathrm{~s}) E_{53} A_{*,-1}^{-1} \Gamma_{2}^{-4}(1+z) & s=2\end{cases}
$$

(e.g., Panaitescu \& Kumar 2000). In these expressions, $\Gamma$ is the Lorentz factor of the shocked fluid, $z$ is the redshift, and we have adopted the usual notation $Q_{n} \equiv Q / 10^{n}$. For $s=2$, the proportionality constant of the density, $A$, is normalized to the typical mass loss rate and stellar wind velocity of a Wolf-Rayet star, which is denoted by $A_{*}$ and is defined as $A_{*} \equiv A /\left(5 \times 10^{11} \mathrm{~g} \mathrm{~cm}^{-1}\right)$ (Chevalier \& Li 2000). For typical GRB afterglow parameters of $E_{53}=1, n_{0}=1$ (or $A_{*}=0.1$ for $s=2), \Gamma_{2}=3$, and $z=2.5$, the deceleration time is under $100 \mathrm{~s}$ for both $s=0$ and $s=2$. Although there can be a large uncertainty in the afterglow parameters $E$ and $n, \Gamma$ is the most important parameter when calculating $t_{\mathrm{dec}}$ since $t_{\mathrm{dec}}$ has a very strong dependence on $\Gamma$. For $s=0(s=2)$, even if we take extreme parameters for $E$ and $n$, such as a high kinetic energy of $E_{53}=100$ and a low density of $n=10^{-3} \mathrm{~cm}^{-3}\left(A_{*}=10^{-2}\right)$, with a typical $\Gamma_{2}=3-4$ (e.g., Molinari et al. 2007; Xue et al. 2009; Liang et al. 2010), $t_{\mathrm{dec}}$ is still a few hundred seconds. Thus, since the deceleration time is less than the typical time at which the steep decline ends, the onset of the external-forward shock emission occurs before the EoSD.

Observationally, the deceleration time is also seen to occur before the EoSD for many GRBs. If the dominant contribution to the light curves at early times is the external-forward shock, the light curve is expected to rise as a power law, reach a peak, and then decline as a power law, with the peak signifying the deceleration time. In Liang et al. 2010, a sample of optical light curves that display this peak is studied. In their Figure 1, for each GRB, they display both the optical light curve and the $\mathrm{X}$-ray light curve. For all their bursts that display a steep decline in the X-ray light curve (except for GRB 080303 and GRB 081203A), it can be seen that the peak of the optical light curve occurs before the end of the X-ray steep decline. We did not include GRB 080330 and GRB 081203A in our samples.

Since an increase in $\epsilon_{B}$ increases $f_{\mathrm{ES}}$ (external-forward shock flux), the condition that the X-ray flux during the steep decline should be larger than or equal to $f_{\mathrm{ES}}$ gives an upper limit on $\epsilon_{B}$. Since our goal is to attain the most stringent upper limit on $\epsilon_{B}$, we take this constraint at the EoSD. Explicitly, the constraint we will use to find an upper limit on $\epsilon_{B}$ with X-ray data is

$$
f_{\mathrm{EoSD}} \geqslant f_{\mathrm{ES}}\left(E, n, s, \epsilon_{e}, \epsilon_{B}, p\right) .
$$

In this inequality, $f_{\text {EoSD }}$ represents the observed flux at the EoSD. We have also explicitly shown the dependence of $f_{\mathrm{ES}}$ on the afterglow parameters. We will now discuss the assumptions we make on the other afterglow parameters, which will allow us to calculate an upper limit on $\epsilon_{B}$.

\subsection{The Other Afterglow Parameters}

\subsubsection{E and $\epsilon_{e}$}

Although we do not know the isotropic equivalent kinetic energy of the blast wave $E$, we can calculate the isotropic energy released in gamma rays during the prompt emission:

$$
E_{\text {iso }}^{\gamma}=\frac{\text { fluence } \times 4 \pi d_{L}^{2}}{1+z} .
$$

In this equation, the fluence has units of $\mathrm{erg} \mathrm{cm}^{-2}$ and represents the flux detected in gamma rays, integrated over the duration of the prompt emission. $d_{L}$ is the luminosity distance. Since we are interested in the fluence radiated in gamma rays, we use the fluence detected in the BAT band, ranging from 15 to $150 \mathrm{keV}$ (Barthelmy et al. 2005). The fluences detected by BAT for each GRB can be found in NASA's Swift GRB Table and Lookup Web site. ${ }^{5}$

To convert $E_{\text {iso }}^{\gamma}$ to $E$, we need to know the efficiency in the conversion of kinetic energy of the jet to prompt gamma-ray emission. Recent studies on the prompt emission efficiency, using X-ray light curves with plateaus detected by Swift, were presented in Granot et al. (2006) and Zhang et al. (2007a). For the 23 GRBs for which Zhang et al. (2007a) presented results for the efficiency (see their Table 3), more than half of them were found to have a high efficiency $\gtrsim 30 \%,{ }^{6}$ with a few being estimated to have an efficiency as high as $90 \%$. A high efficiency of $\sim 90 \%$ was also found in Granot et al. (2006). However, Fan \& Piran (2006) argue that bursts with $\mathrm{X}$-ray plateaus should have more moderate efficiencies $\sim 10 \%$. In addition, Zhang et al. (2007a) mention that the efficiencies they calculate for some bursts have large errors due to the uncertainty in the microphysical parameters $\epsilon_{e}$ and $\epsilon_{B}$. In this work, we will take a standard choice and calculate $E$ with the expression

$$
E=5 E_{\text {iso }}^{\gamma} \text {. }
$$

From the definition of the efficiency, $\eta=E_{\text {iso }}^{\gamma} /\left(E_{\text {iso }}^{\gamma}+E\right)$, Equation (5) corresponds to an efficiency of $\sim 20 \%$. At the end of Section 3.5, we will discuss in more detail how the uncertainty in the efficiency affects our results. For our X-ray sample (see Section 3.3), from Equation (5), we found values for $E$ in the range of $10^{51}-10^{54} \mathrm{erg}$, with a typical value $\sim 10^{53} \mathrm{erg}$. The average value of $E_{53}$ for our X-ray sample is 2.8 , and the median is 1.6. The fluence detected in the BAT band, $E_{\text {iso, } 52}^{\gamma}$, $z$, and $d_{L 28}$ for each GRB in our X-ray sample are shown in Table 1.

For $\epsilon_{e}$, we assumed a value of 0.2 for all of the GRBs in our sample. This choice for $\epsilon_{e}$ is justified from the results of $\epsilon_{e}$ with previous afterglow studies (Figure 1) and with recent results from simulations (Sironi \& Spitkovsky 2011), as discussed in Section 2.

\subsubsection{Electron Power-law Index and Density Profile}

In afterglow studies, $s$ and $p$ can be obtained by determining which closure relation the observed temporal and spectral decay indices satisfy. We cannot use this strategy to determine $p$ and $s$ for our X-ray sample since the external-forward shock flux is below the observed steep decline emission. Instead, we use a fixed $p$ for all GRBs in our X-ray sample. We consider a small value of $p=2.2$, a typical value of $p=2.4$ for Swift GRBs (Curran et al. 2010), and a larger value of $p=2.8$. Previous afterglow studies have found that the majority of afterglow observations are better described by a constant density medium

\footnotetext{
5 http://heasarc.gsfc.nasa.gov/docs/swift/archive/grb_table/

6 In Table 3 of Zhang et al. (2007a), they present two different estimates for the efficiency. If the shallow decay of plateaus seen in X-ray light curves is due to energy injection, the more appropriate of the two estimates for the efficiency is denoted as $\eta_{\gamma}\left(t_{\mathrm{dec}}\right) . \eta_{\gamma}\left(t_{\mathrm{dec}}\right)$ represents the efficiency in gamma-ray radiation $\left(\eta_{\gamma}\right)$ calculated at the deceleration time $t_{\mathrm{dec}}$.

7 Except for GRBs 060708, 060906, 061021, 061222A, 080906, and 081230, we obtained all the redshifts from NASA's Swift GRB Table and Lookup Web site. For the exceptions, we obtained the redshifts from the Web site on GRB redshifts maintained by J. Greiner, http://www.mpe.mpg.de/ jeg/grbgen.html.
} 
Table 1

Properties of X-Ray Sample

\begin{tabular}{|c|c|c|c|c|c|c|c|c|}
\hline GRB & $z$ & $d_{L 28}$ & $\begin{array}{c}\text { Fluence } \\
\left(\times 10^{-6} \mathrm{erg} \mathrm{cm}^{-2}\right)\end{array}$ & $E_{\mathrm{iso}, 52}^{\gamma}$ & $t_{2, \mathrm{EoSD}}$ & $\begin{array}{c}f_{1 \mathrm{keV}, \mathrm{EoSD}} \\
(\mu \mathrm{Jy})\end{array}$ & $\begin{array}{c}\log _{10}\left(\epsilon_{B}\right) \\
(s=0)\end{array}$ & $\begin{array}{c}\log _{10}\left(\epsilon_{B}\right) \\
(s=2)\end{array}$ \\
\hline 050315 & 1.949 & 4.71 & 3.22 & 3.04 & 4 & 3 & -5.2 & -6.0 \\
\hline 050401 & 2.9 & 7.67 & 8.22 & 15.56 & 2 & 80 & -4.7 & -5.4 \\
\hline 050721 & 2.5 & 6.40 & 3.62 & 5.32 & 4 & 30 & -4.2 & -5.0 \\
\hline 050803 & 0.422 & 0.71 & 2.15 & 0.10 & 3 & 10 & -3.8 & -5.4 \\
\hline 050814 & 5.3 & 15.76 & 2.01 & 9.95 & 9 & 1 & -5.4 & -6.0 \\
\hline 051221A & 0.547 & 0.97 & 1.15 & 0.09 & 6 & 5 & -3.5 & -4.9 \\
\hline 060108 & 2.03 & 4.95 & 0.37 & 0.38 & 6 & 0.8 & -4.2 & -5.5 \\
\hline 060111B & 2 & 4.86 & 1.60 & 1.58 & 2 & 8 & -4.6 & -5.8 \\
\hline 060115 & 3.53 & 9.72 & 1.71 & 4.48 & 8 & 1 & -5.2 & -5.9 \\
\hline 060210 & 3.91 & 10.99 & 7.66 & 23.66 & 8 & 40 & -4.4 & -4.7 \\
\hline 060418 & 1.49 & 3.37 & 8.33 & 4.79 & 5 & 40 & -4.3 & -4.9 \\
\hline 060502A & 1.51 & 3.43 & 2.31 & 1.36 & 3 & 7 & -4.6 & -5.7 \\
\hline 060607A & 3.082 & 8.25 & 2.55 & 5.35 & 5 & 60 & -3.6 & -4.3 \\
\hline 060707 & 3.425 & 9.37 & 1.60 & 3.99 & 9 & 3 & -4.5 & -5.2 \\
\hline 060708 & 1.92 & 4.62 & 0.49 & 0.45 & 2 & 10 & -3.7 & -5.2 \\
\hline 060714 & 2.71 & 7.06 & 2.83 & 4.78 & 3 & 10 & -4.8 & -5.7 \\
\hline 060729 & 0.54 & 0.96 & 2.61 & 0.20 & 6 & 7 & -3.8 & -5.1 \\
\hline 060814 & 0.84 & 1.65 & 14.60 & 2.71 & 8 & 8 & -5.0 & -5.6 \\
\hline 060906 & 3.686 & 10.24 & 2.21 & 6.21 & 4 & 0.8 & -5.9 & -6.7 \\
\hline 060926 & 3.208 & 8.66 & 0.22 & 0.49 & 2 & 4 & -3.8 & -5.4 \\
\hline 060927 & 5.6 & 16.81 & 1.13 & 6.08 & 0.8 & 8 & -5.3 & -6.6 \\
\hline 061021 & 0.3463 & 0.56 & 2.96 & 0.09 & 3 & 10 & -3.9 & -5.5 \\
\hline 061110A & 0.758 & 1.45 & 1.06 & 0.16 & 5 & 5 & -3.6 & -6.0 \\
\hline 061121 & 1.314 & 2.88 & 13.70 & 6.19 & 2 & 40 & -5.1 & -5.8 \\
\hline 061222A & 2.088 & 5.13 & 7.99 & 8.55 & 2 & 40 & -4.9 & -5.7 \\
\hline 070110 & 2.352 & 5.94 & 1.62 & 2.14 & 4 & 8 & -4.3 & -5.3 \\
\hline 070306 & 1.497 & 3.39 & 5.38 & 3.12 & 7 & 4 & -5.0 & -5.6 \\
\hline 070714B & 0.92 & 1.85 & 0.72 & 0.16 & 4 & 6 & -3.5 & -5.0 \\
\hline 070802 & 2.45 & 6.24 & 0.28 & 0.40 & 5 & 0.8 & -4.2 & -5.5 \\
\hline 071122 & 1.14 & 2.41 & 0.58 & 0.20 & 8 & 0.8 & -4.1 & -5.4 \\
\hline 080310 & 2.43 & 6.18 & 2.30 & 3.22 & 10 & 2 & -4.8 & -5.4 \\
\hline 080413A & 2.433 & 6.19 & 3.50 & 4.91 & 2 & 10 & -5.1 & -6.0 \\
\hline 080430 & 0.767 & 1.47 & 1.20 & 0.19 & 2 & 10 & -3.9 & -5.5 \\
\hline 080607 & 3.036 & 8.10 & 24.00 & 49.07 & 3 & 90 & -5.2 & -5.5 \\
\hline 080721 & 2.591 & 6.68 & 12.00 & 18.75 & 0.7 & 900 & -4.3 & -5.1 \\
\hline 080905B & 2.374 & 6.00 & 1.80 & 2.42 & 2 & 20 & -4.3 & -5.4 \\
\hline 080906 & 2.1 & 5.16 & 3.50 & 3.78 & 7 & 20 & -4.0 & -4.7 \\
\hline 080916A & 0.689 & 1.29 & 4.00 & 0.50 & 3 & 10 & -4.4 & -5.7 \\
\hline 081007 & 0.5295 & 0.93 & 0.71 & 0.05 & 2 & 8 & -3.5 & -5.3 \\
\hline 081008 & 1.9685 & 4.77 & 4.30 & 4.14 & 6 & 20 & -4.2 & -4.9 \\
\hline 081230 & 2 & 4.86 & 0.82 & 0.81 & 3 & 6 & -4.1 & -5.3 \\
\hline 090418A & 1.608 & 3.71 & 4.60 & 3.05 & 2 & 20 & -4.8 & -5.8 \\
\hline 090516A & 4.109 & 11.66 & 9.00 & 30.08 & 6 & 10 & -5.4 & -5.7 \\
\hline 090519 & 3.85 & 10.79 & 1.20 & 3.62 & 5 & 0.8 & -5.4 & -6.2 \\
\hline 090529 & 2.625 & 6.79 & 0.68 & 1.09 & 20 & 0.5 & -4.3 & -5.1 \\
\hline 090618 & 0.54 & 0.96 & 105.00 & 7.86 & 4 & 200 & -4.9 & -5.3 \\
\hline 090926B & 1.24 & 2.68 & 7.30 & 2.95 & 5 & 10 & -4.8 & -5.6 \\
\hline 091029 & 2.752 & 7.19 & 2.40 & 4.16 & 6 & 1 & -5.5 & -6.2 \\
\hline 091109A & 3.076 & 8.25 & 1.60 & 3.35 & 5 & 2 & -5.0 & -5.8 \\
\hline $100302 \mathrm{~A}$ & 4.813 & 14.06 & 0.31 & 1.33 & 8 & 1 & -4.2 & -5.2 \\
\hline $100425 \mathrm{~A}$ & 1.755 & 4.14 & 0.47 & 0.37 & 3 & 7 & -3.6 & -5.0 \\
\hline $100513 \mathrm{~A}$ & 4.772 & 13.92 & 1.40 & 5.91 & 9 & 7 & -4.1 & -4.8 \\
\hline $100621 \mathrm{~A}$ & 0.542 & 0.96 & 21.00 & 1.58 & 4 & 20 & -5.0 & -5.8 \\
\hline $100704 \mathrm{~A}$ & 3.6 & 9.95 & 6.00 & 16.23 & 6 & 9 & -5.1 & -5.5 \\
\hline $100814 \mathrm{~A}$ & 1.44 & 3.23 & 9.00 & 4.85 & 6 & 9 & -5.0 & -5.6 \\
\hline 100906A & 1.727 & 4.05 & 12.00 & 9.09 & 3 & 20 & -5.2 & -5.9 \\
\hline 110808A & 1.348 & 2.98 & 0.33 & 0.16 & 3 & 3 & -3.6 & -5.2 \\
\hline 110818A & 3.36 & 9.16 & 4.00 & 9.67 & 20 & 1 & -5.3 & -5.5 \\
\hline 111008A & 4.9898 & 14.68 & 5.30 & 23.95 & 3 & 9 & -5.5 & -6.1 \\
\hline $111228 \mathrm{~A}$ & 0.72 & 1.36 & 8.50 & 1.15 & 5 & 8 & -4.8 & -5.7 \\
\hline
\end{tabular}

Notes. This table displays the properties of our X-ray sample. The GRBs that are in bold are also part of our optical sample. The second and third columns show the redshift and the luminosity distance $d_{L}$ (in units of $10^{28} \mathrm{~cm}$ ), respectively. The fourth column shows the fluence detected by BAT in units of $10^{-6} \mathrm{erg} \mathrm{cm}^{-2}$. The next column shows $E_{\text {iso, } 52}^{\gamma}$, the isotropic equivalent energy released in gamma rays during the prompt emission, in units of $10^{52} \mathrm{erg}$. $t_{2, \text { EoSD }}$ represents the time at the end of the steep decline (EoSD) in units of $10^{2} \mathrm{~s}$. The column $f_{1 \mathrm{keV} \text {,EoSD }}$ shows the specific flux at $1 \mathrm{keV}$ at the end of the steep decline, in units of $\mu \mathrm{Jy}$. The last two columns show the upper limits on $\epsilon_{B}$, assuming $p=2.4$. One column shows the results for a constant density medium $(s=0)$ assuming $n=1 \mathrm{~cm}^{-3}$ (filled-in histogram in the top right panel of Figure 2), and the other column shows the results for a wind medium $(s=2)$ assuming $A_{*}=0.1$ (un-filled histogram in the top right panel of Figure 2). 
(e.g., Panaitescu \& Kumar 2002; Schulze et al. 2011). However, there are still a number of cases where the wind medium is a better model for the afterglow observations. Therefore, we will consider both $s=0$ and $s=2$ when displaying the results for the upper limit on $\epsilon_{B}$.

\subsubsection{Density}

The density of the medium in the vicinity of GRBs is a highly uncertain parameter. A histogram of values of $n$ determined by previous afterglow modeling studies can be found in Figure 9 of Soderberg et al. (2006), which shows $n$ to vary over five orders of magnitude, ranging from $\sim 10^{-3} \mathrm{~cm}^{-3}$ to $\sim 10^{2} \mathrm{~cm}^{-3}$. In Section 3.5, we will discuss in more detail how the uncertainty in the density affects our results for the $\epsilon_{B}$ upper limits.

\subsection{The X-Ray Sample}

For our constraint on $\epsilon_{B}$ with X-ray data, we only consider X-ray data detected by the XRT on board Swift. We used the X-ray light curves presented in Butler \& Kocevski (2007). ${ }^{8}$ With the exception of two cases, we only consider bursts that display a steep decline in their X-ray light curve (see below). After the EoSD, GRBs display a variety of temporal decays (Evans et al. 2009). Our sample can be divided into four different subgroups, based on the temporal decay after the steep decline.

1. Steep decline to plateau. In this subgroup, GRBs display a plateau after the steep decline ( $\sim 73 \%$ of our sample). In Table 1, we display the time at the EoSD in units of $10^{2} \mathrm{~s}, t_{2, \text { EoSD }}$, and the observed flux at the EoSD at $1 \mathrm{keV}$, $f_{1 \mathrm{keV}, \mathrm{EoSD}}$, in $\mu \mathrm{Jy}$.

2. Steep decline to normal decline. In this subgroup, GRBs display a temporal decay of $\alpha \sim 1$ after the steep decline ( $\sim 18 \%$ of our sample). In Table 1 , we show the time and the flux at the EoSD.

3. Clear steep decline but not a clear end to the steep decline. In this subgroup, it cannot be determined where the steep decline ends (the XRT observations end before the steep decline ends). The following GRBs fall into this subgroup: 050315, 060202, 070419A, 071122, and 090516. For these GRBs, in Table 1, we show the flux and the time of the last steep decline point observed. For these GRBs, to be sure that the last steep decline point observed is past the deceleration time, we made sure that it is at a few hundred seconds.

4. No clear steep decline seen, just plateau. Two of the GRBs in our X-ray sample, 050401 and 060927, do not display a steep decline. The first observation of the X-ray light curve for these bursts is during the plateau. We did not remove these GRBs from our X-ray sample because they are also part of our optical sample (bursts with both X-ray and optical data are important because they allow us to cross-check our results; see Section 5). For these two bursts, we considered the first observation in the X-ray light curve for our constraint so that we have the least amount of energy injection. We made sure that this point is at least at a few hundred seconds so that we can be confident that the onset of the external-forward shock emission has occurred. For these two bursts, we show the time and the flux of the first $\mathrm{X}$-ray observation of the plateau in Table 1.

In addition, $25 \%$ of the GRBs in our X-ray sample display $\mathrm{X}$-ray flares during the steep decline. We only consider bursts where the X-ray flare ends before the EoSD because it is difficult

\footnotetext{
8 http://butler.lab.asu.edu/swift/
}

to determine the flux and time at the EoSD for bursts that show $\mathrm{X}$-ray flares near the EoSD. It is fine to consider these bursts because observationally, after the X-ray flare, the X-ray light curve is seen to return to the same temporal decay prior to the X-ray flare (e.g., Chincarini et al. 2007). Lastly, GRB 051221A is the only short GRB in our X-ray sample; all the other bursts in our X-ray sample are long GRBs.

\subsection{Expected External-forward Shock Emission at the End of the Steep Decline}

The synchrotron afterglow spectrum consists of four powerlaw segments that are smoothly joined together at three characteristic frequencies of synchrotron emission (e.g., Sari et al. 1998; Granot \& Sari 2002). These three characteristic frequencies are: $v_{a}$, the synchrotron self-absorption frequency, $v_{i}$ (also commonly referred to as $v_{m}$ ), the frequency of the photons emitted by the power-law distribution of injected electrons with the smallest energy, and $v_{c}$, the cooling frequency corresponding to electrons cooling on a dynamical time. For this work, we will only consider the standard case for the ordering of the characteristic frequencies, the slow cooling case, where $v_{a}<v_{i}<v_{c}$. One argument against the fast cooling case $\left(v_{a}<v_{c}<v_{i}\right)$ is that if the observing frequency is between $v_{c}$ and $v_{i}$, the spectrum should be $f_{v} \propto v^{-1 / 2}$; however, the spectral index $\beta=0.5$ disagrees with the typical observed afterglow spectral index $\beta \approx 0.9$ (e.g., Piro 2001). The next question we need to consider is where the X-ray band lies at the EoSD (here, we consider $1 \mathrm{keV}$ for the $\mathrm{X}$-ray band because the light curves we used are plotted at this energy). The two possibilities for the spectral regime of the $\mathrm{X}$-ray band are $v_{i}<1 \mathrm{keV}<v_{c}$ or $v_{c}<1 \mathrm{keV}$. We rule out $v_{c}<1 \mathrm{keV}$ with the following two arguments.

First, we compare the observed flux at the EoSD, $f_{1 \mathrm{keV} \text {,EoSD, }}$ to the flux predicted by the external-forward shock at the same time, if $v_{c}<1 \mathrm{keV}$ (defined as $f_{\text {pred }}$ ). For $s=0, f_{\text {pred }}$ is given by (Granot \& Sari 2002)

$$
\begin{aligned}
f_{\text {pred }}= & 0.855(p-0.98) 10^{\frac{3 p+2}{4}} 8.64^{\frac{3 p-2}{4}} e^{1.95 p} \\
& \times(1+z)^{\frac{p+2}{4}}\left(\bar{\epsilon}_{e,-1}\right)^{p-1} \epsilon_{B}^{\frac{p-2}{4}} E_{53}^{\frac{p+2}{4}}\left(t_{2, \text { EoSD }}\right)^{-\frac{(3 p-2)}{4}} \\
& \times d_{L 28}^{-2} v_{14}^{-\frac{p}{2}} \mathrm{mJy},
\end{aligned}
$$

where $\bar{\epsilon}_{e,-1} \equiv(p-2) /(p-1) \epsilon_{e,-1}$. For $v_{c}<1 \mathrm{keV}$, the external-forward shock flux is independent of the density and the $s=2$ expression is almost identical. When calculating $f_{\text {pred }}$, for each of the bursts in our X-ray sample, we assumed a standard $p=2.4, \epsilon_{e}=0.2$, and $v_{14}=2.4 \times 10^{3}$, the frequency corresponding to $1 \mathrm{keV}$. For the parameters $E, t, z$, and $d_{L}$, we used the values given in Table 1 for each burst $\left(E=5 E_{\text {iso }}^{\gamma}\right)$. The remaining parameter we need to compute $f_{\text {pred }}$ is $\epsilon_{B}$. Since $\epsilon_{B}$ is raised to the power of $(p-2) / 4$, for a typical $p \sim 2-3$, the dependence on $\epsilon_{B}$ is very weak. When calculating $f_{\text {pred }}$, we assumed a low value of $\epsilon_{B}=10^{-3}$.

We computed the ratio $f_{\text {pred }} / f_{1 \mathrm{keV}, \text { EoSD }}$ for all the GRBs in our X-ray sample and found that $f_{\text {pred }} / f_{1 \mathrm{keV} \text {,EoSD }}>1$ for all the bursts and $f_{\text {pred }} / f_{1 \mathrm{keV} \text {,EoSD }}>10$ for $54 / 60$ bursts. The mean value of $f_{\text {pred }} / f_{1 \mathrm{keV} \text {,EoSD }}$ is 50 , and the median value is 34 . This means that the predicted flux from the external-forward shock, when $v_{c}<1 \mathrm{keV}$, overpredicts the observed flux at the end of the steep decline by a factor that is larger than 10 for the majority of the bursts. Therefore, the assumption that $v_{c}<1 \mathrm{keV}$ is incorrect. This is a robust conclusion because the $\mathrm{X}$-ray flux from the external-forward shock, when $v_{c}<1 \mathrm{keV}$, 
basically only depends on $\sim \epsilon_{e} E$ (see Equation (6)), which cannot be decreased by a factor of $>10$ without introducing serious efficiency problems in producing the prompt gammarays. Even if we allow for an uncertainty of a factor of $\sim 2-3$ in both $\epsilon_{e}$ and $E$, this is not enough to decrease $f_{\text {pred }} / f_{1 \mathrm{keV} \text {,EoSD }}$ below 1 for the majority of bursts in our X-ray sample.

Before continuing, we want to add that $f_{\text {pred }}$ (Equation (6)) also has a dependence on the Compton- $Y$ parameter: $f_{\text {pred }} \propto$ $(1+Y)^{-1}$. With this dependence, if the Compton- $Y$ parameter is large, then it is possible for $f_{\text {pred }}$ to decrease below $f_{1 \mathrm{keV} \text {,EoSD }}$. For a few bursts in our X-ray sample, we performed a detailed numerical calculation of the external-forward shock flux with the formalism presented in Barniol Duran \& Kumar (2011), which includes a detailed treatment of Compton- $Y$ with KleinNishina effects. From this calculation, we also found that $f_{\text {pred }}$ overpredicts $f_{1 \mathrm{keV} \text {,EoSD }}$ by a factor larger than 10 , which means that the inverse Compton (IC) cooling of electrons producing $1 \mathrm{keV}$ synchrotron photons is a weak effect (even when $\epsilon_{B}$ is small). Numerically, we also found that without making any assumption about the location of $v_{c}$, solutions to the constraint $f_{\mathrm{ES}, 1 \mathrm{keV}} \leqslant f_{1 \mathrm{keV} \text {,EoSD }}$ were only found when $1 \mathrm{keV}<v_{c}$ (when $\epsilon_{B}$ is small such that $1 \mathrm{keV}<v_{c}$, it turns out that IC cooling of electrons producing $1 \mathrm{keV}$ photons takes place in the KleinNishina regime at these early times of $\sim$ few $\times 100 \mathrm{~s}$ ).

Another argument in favor of the spectral regime of the X-ray band being $v_{i}<1 \mathrm{keV}<v_{c}$ at the EoSD comes from the extrapolation of $v_{c}$ at late times to the EoSD. In Liang et al. (2008), they made fits to late-time XRT light curves during the normal decline phase and also provided the value of $v_{c}$ during the geometrical midpoint of the normal decline phase. ${ }^{9}$ For this argument, we focus on GRBs that are in common with our X-ray sample and the sample of Liang et al. (2008). For these bursts, we extrapolate $v_{c}$ at late times to the EoSD. In Liang et al. (2008), they only considered a constant density medium, where $v_{c} \propto t^{-1 / 2}$. The results of the extrapolation of $v_{c}$ are shown in Table 2. In Table 2, we find that at the EoSD, $1 \mathrm{keV}<v_{c \text {, EoSD }}$ for all GRBs. This further confirms our choice that $v_{i}<1 \mathrm{keV}<v_{c}$ at the EoSD. ${ }^{10}$

The knowledge of the spectral regime at the EoSD allows us to write in an explicit expression for $f_{\mathrm{ES}}$ in Equation (3):

$$
\frac{f_{1 \mathrm{keV}, \mathrm{EoSD}}}{\mathrm{mJy}} \geqslant\left\{\begin{array}{c}
0.461(p-0.04) 10^{\frac{3 p+1}{4}} 8.64^{\frac{3(p-1)}{4}} e^{2.53 p} \\
\times(1+z)^{\frac{p+3}{4}}\left(\bar{\epsilon}_{e,-1}\right)^{p-1} \epsilon_{B}^{\frac{p+1}{4}} n_{0}^{\frac{1}{2}} E_{53}^{\frac{p+3}{4}} \\
\times\left(t_{2, \mathrm{EoSD}}\right)^{-\frac{3(p-1)}{4}} d_{L 28}^{-2} v_{14}^{-\frac{(p-1)}{2}} s=0 \\
3.82(p-0.18) 10^{\frac{3 p-1}{4}} 8.64^{\frac{3 p-1}{4}} e^{2.54 p} \\
\times(1+z)^{\frac{p+5}{4}}\left(\bar{\epsilon}_{e,-1}\right)^{p-1} \epsilon_{B}^{\frac{p+1}{4}} A_{*,-1} E_{53}^{(p+1) / 4} \\
\times\left(t_{2, \mathrm{EoSD}}\right)^{-\frac{(3 p-1)}{4}} d_{L 28}^{-2} v_{14}^{-\frac{(p-1)}{2}} s=2 .
\end{array}\right.
$$

\footnotetext{
9 The geometrical midpoint of the normal decline is defined by $\log _{10} t=\left(\log _{10} t_{1}+\log _{10} t_{2}\right) / 2$, where $t_{1}$ represents the time of the first observation of the normal decline and $t_{2}$ represents the time of the last observation of the normal decline.

10 Including energy injection will make the values of $v_{\mathrm{c}, \text { EoSD }}$ in Table 2 larger, making the conclusion that $v_{i}<1 \mathrm{keV}<v_{c}$ at the end of the steep decline more robust. For the bursts that have plateaus in their X-ray light curve, energy injection needs to be considered. During the energy injection episode, $E$ increases as $E \propto t^{1-q}$ (Zhang et al. 2006), where $q$ is a positive constant that satisfies $0 \leqslant q \leqslant 1$. Therefore, between the end of the plateau and the end of the steep decline, since $v_{c} \propto E^{-1 / 2} t^{-1 / 2}, v_{c} \propto t^{-1+(q / 2)}$. This time evolution of $v_{c}$ is steeper than $v_{c} \propto t^{-1 / 2}$ without energy injection for $s=0$ (Liang et al. 2008 only considered $s=0$ ).
}

Table 2

Extrapolation of $v_{c}$ from the Normal Decline to the EoSD

\begin{tabular}{lrccc}
\hline \hline GRB & $t_{\text {late }, 4}$ & $t_{\text {EoSD }, 2}$ & $\begin{array}{l}v_{\mathrm{c}, \text { late }} \\
(\mathrm{keV})\end{array}$ & $\begin{array}{c}v_{\mathrm{c}, \text { EoSD }} \\
(\mathrm{keV})\end{array}$ \\
\hline 050315 & 3.5 & 4 & 0.17 & 1.6 \\
050401 & 6.9 & 2 & 4.1 & 77 \\
$051221 \mathrm{~A}$ & 1.7 & 6 & 4.1 & 22 \\
060210 & 3.2 & 8 & 0.97 & 6.1 \\
$060502 \mathrm{~A}$ & 0.42 & 3 & 4.1 & 15 \\
060714 & 3.5 & 3 & 3.6 & 39 \\
060729 & 40 & 6 & 1.4 & 36 \\
060814 & 2.0 & 8 & 4.1 & 21 \\
060926 & 0.26 & 2 & 6.7 & 24 \\
061121 & 1.1 & 2 & 4.2 & 31 \\
070110 & 14 & 4 & 4 & 74 \\
\hline
\end{tabular}

Notes. This table shows the extrapolation of $v_{c}$ from late times to the end of the steep decline for GRBs in common to our sample and to the sample in Liang et al. (2008; first column). The second column shows $t_{\text {late }, 4}$, the late time in units of $10^{4} \mathrm{~s}$ at which Liang et al. (2008) determined $v_{c}$. The third column shows $t_{\mathrm{EoSD}, 2}$, the time at the end of the steep decline in units of $10^{2} \mathrm{~s} . v_{\mathrm{c}, \text { late }}$, given in $\mathrm{keV}$, is the value found in Liang et al. (2008) for $v_{c}$ at $t_{\text {late. The last }}$ column shows $v_{\mathrm{c}, \text { EoSD }}$ in keV. $v_{\mathrm{c}, \text { EoSD }}$ is found by extrapolating $v_{\mathrm{c} \text {, late }}$ to $t_{\mathrm{EoSD}}$. Since Liang et al. (2008) assume a constant density medium, we take a constant density medium for all the GRBs in this sample when making the extrapolation of $v_{c}$ to $t_{\mathrm{EoSD}}$.

On the left-hand side of this inequality, we have the observed $\mathrm{X}$-ray flux at the EoSD, and on the right-hand side we have the expression for the external-forward shock flux when $v_{i}<$ $1 \mathrm{keV}<v_{c}$ (Granot \& Sari 2002). The notation used for $\bar{\epsilon}_{e}$ and $A_{*}$ is defined as $\bar{\epsilon}_{e,-1} \equiv \bar{\epsilon}_{e} / 10^{-1}$ and $A_{*,-1} \equiv A_{*} / 10^{-1}$. The expressions in Equation (7) are only valid for $p>2$, which we consider in this work (for $p<2$, see Bhattacharya 2001; Resmi \& Bhattacharya 2008).

Before displaying our results for the $\epsilon_{B}$ upper limit for our entire X-ray sample, we show a simple calculation to get an idea of what values to expect for the $\epsilon_{B}$ upper limits from the X-ray constraint given in Equation (7). For a standard $p=2.4$, the $\mathrm{X}$-ray constraint is

$$
\frac{f_{1 \mathrm{keV}, \mathrm{EoSD}}}{\mathrm{mJy}} \geqslant \begin{cases}5.0 \times 10^{1} t_{2, \mathrm{EoSD}}^{-1.05} \epsilon_{e,-1}^{1.4} E_{53}^{1.35} n_{0}^{0.5} \epsilon_{B}^{0.85} & s=0 \\ 7.0 \times 10^{2} t_{2, \mathrm{EoSD}}^{-1.55} \epsilon_{e,-1}^{1.4} E_{53}^{0.85} A_{*,-1} \epsilon_{B}^{0.85} & s=2 .\end{cases}
$$

For this calculation, we used the average $z=2.5$ for Swift GRBs (Gehrels et al. 2009; with a corresponding $d_{L 28}=6.4$ ) and $v_{14}$ corresponding to $1 \mathrm{keV}$. Solving for $\epsilon_{B}$, the upper limit depends on the afterglow parameters as ${ }^{11}$

$$
\epsilon_{B} \leqslant\left\{\begin{array}{ccc}
1.0 \times 10^{-2}\left(\frac{f_{1 \mathrm{keV}, \mathrm{EoSD}}}{\mathrm{mJy}}\right)^{1.18} t_{2, \mathrm{EoSD}}^{1.24} & \\
& \times \epsilon_{e,-1}^{-1.65} E_{53}^{-1.59} n_{0}^{-0.59} & s=0 \\
4.5 \times 10^{-4}\left(\frac{f_{1 \mathrm{keV}, \mathrm{EoSD}}}{\mathrm{mJy}}\right)^{1.18} t_{2, \mathrm{EoSD}}^{1.82} & \\
\times \epsilon_{e,-1}^{-1.65} E_{53}^{-1} A_{*,-1}^{-1.18} & & s=2 .
\end{array}\right.
$$

\footnotetext{
${ }^{11}$ For bursts with plateaus in their X-ray light curve, it is possible that energy injection begins before the steep decline ends. However, even with energy injection, there still exists a self-similar solution for the energy (Equation (52) of Blandford \& McKee 1976). For both $s=0$ and $s=2$, we calculated the external-forward shock synchrotron flux with this new self-similar solution and then calculated the upper limit on $\epsilon_{B}$ as shown in Equation (9). We found that the $\epsilon_{B}$ upper limits are affected by less than a factor of $\sim 2$. Thus, even if energy injection begins before the end of the steep decline, it has very little to no effect on our $\epsilon_{B}$ upper limit results.
} 
Table 3

Mean and Median $\epsilon_{B}$ Values for the X-Ray (Upper Limits on $\epsilon_{B}$ ) and Optical (Measurements of $\epsilon_{B}$ ) Histograms Shown in Figures 2 and 3

\begin{tabular}{|c|c|c|c|c|}
\hline $\begin{array}{l}\text { X-ray } \\
(s=0)\end{array}$ & $p=2.2$ & $p=2.4$ & $p=2.8$ & \\
\hline Mean & $1.1 \times 10^{-4}$ & $7.2 \times 10^{-5}$ & $6.1 \times 10^{-5}$ & \\
\hline Median & $4.2 \times 10^{-5}$ & $2.8 \times 10^{-5}$ & $2.0 \times 10^{-5}$ & \\
\hline $\begin{array}{l}\text { X-ray } \\
(s=2)\end{array}$ & $p=2.2$ & $p=2.4$ & $p=2.8$ & \\
\hline Mean & $7.9 \times 10^{-6}$ & $5.5 \times 10^{-6}$ & $5.6 \times 10^{-6}$ & \\
\hline Median & $4.0 \times 10^{-6}$ & $3.2 \times 10^{-6}$ & $3.4 \times 10^{-6}$ & \\
\hline $\begin{array}{l}\text { Opt. } \\
(s=0)\end{array}$ & $p=2.2$ & $p=2.4$ & $p=2.8$ & $p$ from $\alpha_{\mathrm{O}}$ \\
\hline Mean & $3.5 \times 10^{-4}$ & $1.0 \times 10^{-4}$ & $2.9 \times 10^{-5}$ & $9.5 \times 10^{-5}$ \\
\hline Median & $1.1 \times 10^{-4}$ & $3.3 \times 10^{-5}$ & $5.5 \times 10^{-6}$ & $2.4 \times 10^{-5}$ \\
\hline $\begin{array}{l}\text { Opt. } \\
(s=2)\end{array}$ & $p=2.2$ & $p=2.4$ & $p=2.8$ & \\
\hline Mean & $7.2 \times 10^{-5}$ & $2.4 \times 10^{-5}$ & $7.5 \times 10^{-6}$ & \\
\hline Median & $1.2 \times 10^{-5}$ & $3.9 \times 10^{-6}$ & $8.8 \times 10^{-7}$ & \\
\hline
\end{tabular}

Notes. The section labeled "X-ray ( $(=0)$ )" ("X-ray $(s=2)$ )") shows the mean and median $\epsilon_{B}$ upper limits assuming a constant density (wind) medium with a standard $n=1 \mathrm{~cm}^{-3}\left(A_{*}=0.1\right)$. The columns show the value of $p$ that was assumed. The section labeled "Opt. ( $s=0)$ " ("Opt. $(s=2)$ ") shows the mean and median $\epsilon_{B}$ measurements assuming a constant density (wind) medium with a standard $n=1 \mathrm{~cm}^{-3}\left(A_{*}=0.1\right)$. The columns show the value of $p$ that was assumed. The column labeled " $p$ from $\alpha_{O}$ " shows the mean and median $\epsilon_{B}$ measurements with $p$ determined from $\alpha_{O}$. There are $60 \mathrm{GRBs}$ in our X-ray sample and 35 GRBs in our optical sample.

The median values for our X-ray sample for the parameters $f_{1 \mathrm{keV}, \mathrm{EoSD}}, t_{2, \mathrm{EoSD}}$, and $E_{53}$ are $8 \times 10^{-3} \mathrm{mJy}, 4$, and 1.6, respectively. Using these median values and $\epsilon_{e,-1}=2$, the upper limit on $\epsilon_{B}$ becomes

$$
\epsilon_{B} \leqslant \begin{cases}2.8 \times 10^{-5} \times n_{0}^{-0.59} & s=0 \\ 3.7 \times 10^{-6} \times A_{*-1}^{-1.18} & s=2 .\end{cases}
$$

For a standard $n_{0}=1$ and $A_{*,-1}=1$, it can be seen that the $\epsilon_{B}$ upper limit is lower for $s=2$. This is expected because for $A_{*,-1}=1$, there is a larger density for the surrounding medium within a typical deceleration radius of $10^{17} \mathrm{~cm}$.

In Equation (10), the explicit dependence of the $\epsilon_{B}$ upper limit on the density is shown for $p=2.4$. In the next subsection, we will display the results of the $\epsilon_{B}$ upper limits for our entire $\mathrm{X}$-ray sample. To keep the density dependence, we will display histograms of upper limits on the quantity $\epsilon_{B} n_{0}^{0.59}\left(\epsilon_{B} A_{*-1}^{1.18}\right)$ for $s=0(s=2)$ for $p=2.4$, or $\epsilon_{B} n_{0}^{2 /(p+1)}\left(\epsilon_{B} A_{*-1}^{4 /(p+1)}\right)$ for $s=0$ $(s=2)$ for a general $p$ (see Equation (7)).

\section{5. $\epsilon_{B}$ Upper Limits for Our X-Ray Sample}

We display the results for the upper limits (from Equation (7)) on the quantity $\epsilon_{B} n_{0}^{2 /(p+1)}\left(\epsilon_{B} A_{*-1}^{4 /(p+1)}\right)$ for $s=0(s=2)$ assuming that all GRBs in our X-ray sample have $p=2.2$, 2.4, and 2.8 in the top left, top right, and bottom panels of Figure 2, respectively. Two histograms are shown in each panel, one for $s=0$ and the other for $s=2$. Table 3 shows the mean and median upper limits on the quantity $\epsilon_{B} n_{0}^{2 /(p+1)}\left(\epsilon_{B} A_{*-1}^{4 /(p+1)}\right)$ for $s=0(s=2)$ for each histogram. For the remainder of this section, we assume a standard $n_{0}=1\left(A_{*-1}=1\right)$ for $s=0$ $(s=2)$ when discussing our results for the $\epsilon_{B}$ upper limits.
The $\epsilon_{B}$ upper limit histograms show a wide distribution. For a constant density (wind) medium, all the histograms show a distribution in the range $\sim 10^{-6}-10^{-3}\left(\sim 10^{-7}-10^{-4}\right)$. For a constant density (wind) medium, the mean and median $\epsilon_{B}$ upper limit values are $\sim$ few $\times 10^{-5}\left(\sim\right.$ few $\left.\times 10^{-6}\right)$. Assuming a different value of $p$ does not have a significant effect on the distributions of the $\epsilon_{B}$ upper limits for our X-ray sample. For both the $s=0$ and $s=2$ cases, when changing $p$, the mean and median $\epsilon_{B}$ upper limit values change by less than a factor $\sim 2$. Although previous afterglow studies also showed a wide distribution for $\epsilon_{B}$ (Figure 1), our distribution of $\epsilon_{B}$ upper limits is shifted toward lower values. Unlike Figure 1, which shows that many GRBs have been reported to have $\epsilon_{B} \sim 10^{-3}-10^{-1}$, none of our histograms of $\epsilon_{B}$ upper limits show an $\epsilon_{B}$ upper limit larger than $10^{-3}$.

We now discuss how our assumptions on the afterglow parameters can affect the distribution of $\epsilon_{B}$ upper limits. For this discussion, we will take a typical $p=2.4$; Equation (9) shows how the $\epsilon_{B}$ upper limit depends on the other afterglow parameters. The strongest dependence is on $\epsilon_{e}$, which is raised to the power of -1.65 . However, as we displayed in Figure 1, according to previous studies, the distribution of $\epsilon_{e}$ values is narrow, with the $\epsilon_{e}$ values ranging only over one order of magnitude. In addition, $\sim 62 \%$ of the bursts have $\epsilon_{e} \sim 0.1-0.3$. From Figure 1, a likely error in $\epsilon_{e}$ from our assumed $\epsilon_{e}=0.2$ is a factor $\sim 2$. From Equation (9), an error in $\epsilon_{e}$ by a factor $\sim 2$ will only lead to an error in the $\epsilon_{B}$ upper limit by a factor $\sim 3$. For a constant density (wind) medium, the $\epsilon_{B}$ upper limit depends on $E$ as $E^{-1.59}\left(E^{-1}\right)$. We assumed an efficiency of $\sim 20 \%$ in the conversion of kinetic energy to prompt gammaray radiation. Recent studies have found higher values for the efficiency (Granot et al. 2006, Zhang et al. 2007a; see, however, Fan \& Piran 2006). In Zhang et al. (2007a), the mean (median) efficiency they reported is $\sim 37 \%(\sim 32 \%)$. Taking the efficiency to be $\sim 30 \%-40 \%$ instead of $\sim 20 \%$ would lead to an error in $E$ by a factor $\sim 2-3$. From Equation (9), an error in $E$ by a factor $\sim 2-3$ would lead to an error in the $\epsilon_{B}$ upper limit by a factor $\sim 3-6$ $(\sim 2-3)$ for a constant density (wind) medium. Lastly, the largest source of uncertainty for the $\epsilon_{B}$ upper limits is the density, since it has been reported to have a range $\sim 10^{-3} \mathrm{~cm}^{-3}-10^{2} \mathrm{~cm}^{-3}$. For a constant density (wind) medium, the $\epsilon_{B}$ upper limit depends on the density as $n^{-0.59}\left(A_{*}^{-1.18}\right)$. For $s=0(s=2)$, we assumed a standard $n=1 \mathrm{~cm}^{-3}\left(A_{*}=0.1\right)$. An error in the density by a factor $\sim 10^{3}\left(\sim 10^{2}\right)$ will lead to an error in the $\epsilon_{B}$ upper limit by a factor $\sim 60(\sim 230)$.

In summary, the expected errors in $\epsilon_{e}$ and $E$ of a factor $\sim 2-3$ will not change the $\epsilon_{B}$ upper limits by an order of magnitude. On the other hand, the density is a very uncertain parameter and an error in the density by $\sim 2-3$ orders of magnitude will lead to an error in the $\epsilon_{B}$ upper limits by $\sim 1-2$ orders of magnitude.

Two additional parameters that can affect our $\epsilon_{B}$ upper limits are (1) $\xi$, the fraction of electrons accelerated to a power-law distribution, and (2) $f$, which is a factor that takes into account the degeneracy for a set of afterglow parameters. For a set of parameters $E, n, \epsilon_{e}, \epsilon_{B}, \xi$ producing the observed externalforward shock flux, another set of primed parameters $E^{\prime}=E / f$, $n^{\prime}=n / f, \epsilon_{e}^{\prime}=f \epsilon_{e}, \epsilon_{B}^{\prime}=f \epsilon_{B}, \xi^{\prime}=f \xi$ can also produce the observed external-forward shock flux (Eichler \& Waxman 2005). Afterglow studies usually assume $\xi=f=1$ for simplicity but $\xi \leqslant 1$ and $m_{e} / m_{p} \leqslant f \leqslant 1$ (Eichler \& Waxman $2005)$, where $m_{e}\left(m_{p}\right)$ is the electron (proton) mass. The externalforward shock flux depends on $\xi$ and $\epsilon_{B}$ as $f_{\nu} \propto \xi^{2-p} \epsilon_{B}^{(p+1) / 4}$ (Leventis et al. 2012). From this dependence, we find that the 

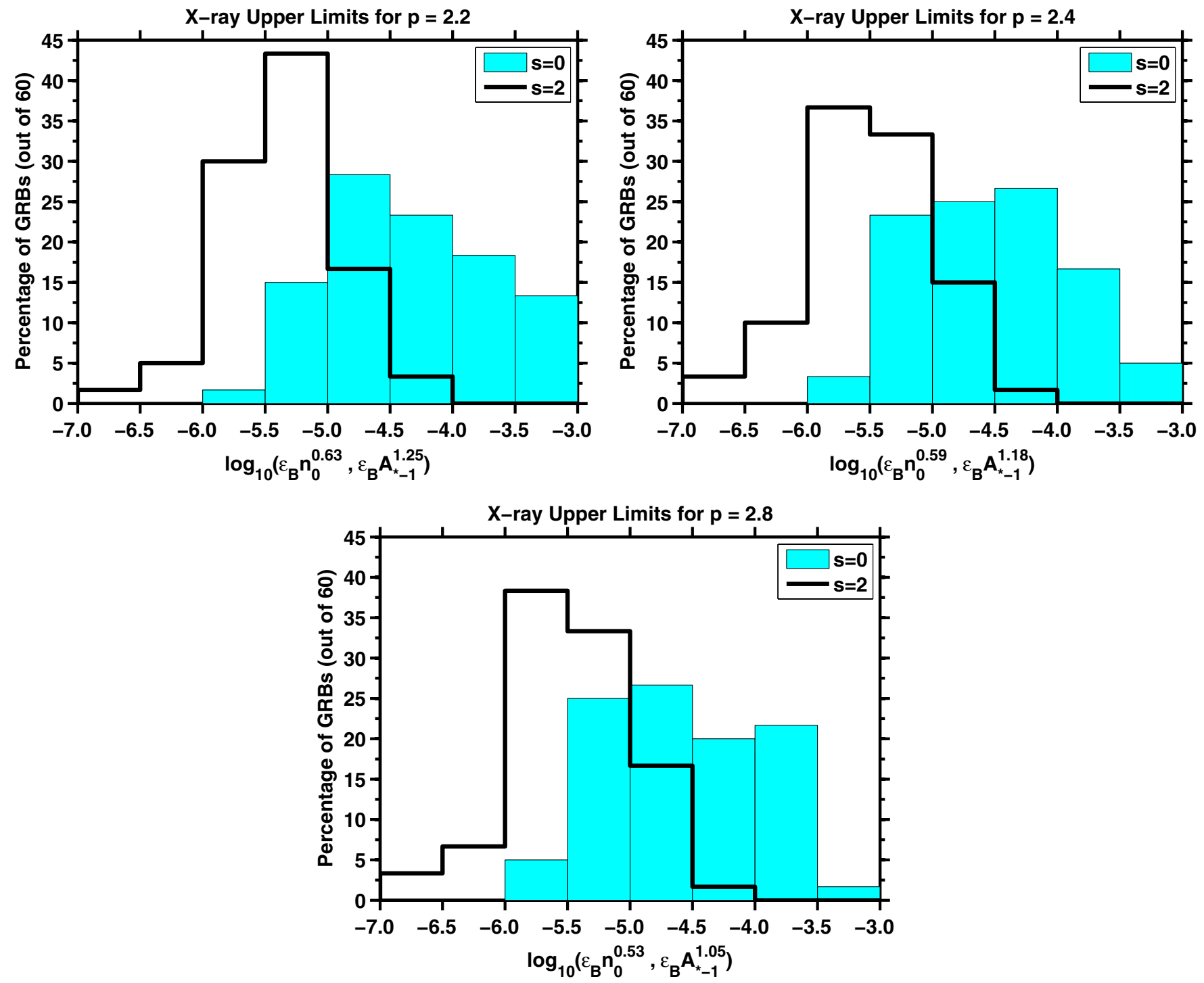

Figure 2. Top left, top right, and bottom panels show the histograms of upper limits assuming $p=2.2, p=2.4$, and $p=2.8$, respectively, for all of the GRBs in our $\mathrm{X}$-ray sample (obtained with Equation (7)). The filled-in (un-filled) histograms show upper limits on the quantity $\epsilon_{B} n_{0}^{2 /(p+1)}\left(\epsilon_{B} A_{*-1}^{4 /(p+1)}\right)$ for $s=0(s=2)$ assuming that all the GRBs in our X-ray sample are described by a constant density (wind) medium.

(A color version of this figure is available in the online journal.)

$\epsilon_{B}$ upper limit $\propto \xi^{4(p-2) /(p+1)}$. Thus, including $\xi$ will decrease the values of our $\epsilon_{B}$ upper limits. Values of $\xi$ have not been determined for GRB external-forward shocks so we cannot quantify by how much the $\epsilon_{B}$ upper limit values will decrease. Including $f$ will also decrease the values of the $\epsilon_{B}$ upper limits since $\epsilon_{B}^{\prime}=f \epsilon_{B}$ and $f<1$. Like $\xi$, values of $f$ have also not been determined from afterglow observations. The largest effect $f$ can have on the $\epsilon_{B}$ upper limit values is decrease them by a factor of $m_{p} / m_{e} \sim 2000$. For the remainder of this paper, we will be conservative and continue to assume $\xi=f=1$, but we should keep in mind that considering $\xi$ and $f$ will decrease the values of the $\epsilon_{B}$ upper limits.

\section{MEASUREMENT OF $\epsilon_{B}$ WITH OPTICAL LIGHT CURVES}

\section{1. $\epsilon_{B}$ Determination with Optical Data}

The light curves we consider for our optical sample decline with a temporal decay index $\alpha \sim 1$ from early times, $\sim 10^{2}-10^{3} \mathrm{~s}$, as expected for the external-forward shock emis- sion (see Section 4.2). Since the light curves of these bursts are likely dominated by the external-forward shock, this means that the observed optical flux is an actual measurement of the external-forward shock flux, that is ${ }^{12}$

$$
f_{\mathrm{obs}}=f_{\mathrm{ES}}\left(E, n, \epsilon_{e}, \epsilon_{B}, p, s\right) .
$$

Later in this section we will use this condition to determine $\epsilon_{B}$ for the bursts in our optical sample. We want to stress that we

\footnotetext{
12 At late times, $\sim 10^{3}-10^{5} \mathrm{~s}$, many X-ray light curves decline with $\alpha \sim 1$ : the "normal" decline. If this segment arises from the external-forward shock, then $\epsilon_{B}$ can be determined as described in this section for our optical sample. However, this is not straightforward since energy injection (as evidenced by the plateau phase) should be considered. Also, one can attempt to use the upper limit on $\epsilon_{B}$, found in Section 3, to calculate a lower limit on $v_{c}$ during the normal decline and compare this to the observed spectral regime $\left(1 \mathrm{keV}<v_{c}\right.$ or $\left.1 \mathrm{keV}>v_{c}\right)$. However, there are two difficulties with this $v_{c}$ consistency check: (1) several "closure relations" are simultaneously satisfied within $2 \sigma$ for most of our sample (due to large errors in the spectral and temporal indices at late times, which can be found in Evans et al. 2007, 2009); and (2) for the bursts that uniquely satisfy one closure relation, $v_{c}$ cannot be determined precisely since Klein-Nishina suppression is weaker at late times and $v_{c}$ depends strongly on $n$ when Compton- $Y$ is considered.
} 
determine $\epsilon_{B}$ for the optical sample. This is in contrast to the $\mathrm{X}$-ray sample, which only allowed us to determine an upper limit on $\epsilon_{B}$.

\subsection{The Optical Sample}

Our optical sample consists of 35 GRBs. A total of 33/35 of the bursts triggered Swift, and the remaining two bursts, 050502A and 080603A, were detected by the International Gamma-Ray Astrophysics Laboratory (INTEGRAL; Winkler et al. 2003). Table 4 shows properties of our optical sample. With a few exceptions, most of the GRBs in our optical sample have a known redshift. ${ }^{13}$

With the exception of the only short GRB in our optical sample, GRB 051221A, all the optical light curves in our sample decline before $3500 \mathrm{~s}$. Considering early times has the advantage of minimizing possible energy injection. Our optical sample can be separated into four different subgroups, depending on the temporal behavior of the light curve before the $\alpha \sim 1$ decay as follows.

1. Light curves with a peak at early times. The light curves of this subgroup are characterized by a power-law rise, reaching a peak, and then a power-law decline with $\alpha_{O} \sim 1$ ( $\sim 43 \%$ of our sample). The peak of the light curve is believed to be due to the deceleration time. For the bursts in this subgroup, we show the temporal decay of the optical light curve after the peak and the flux and the time of the second data point after the peak in Table 4. We take the second data point to be confident that the optical light curve is declining.

2. Single power-law decay from early times. In this subgroup, the optical light curve shows a decline as a single power law with $\alpha \sim 1$ from the beginning of the observations ( $\sim 40 \%$ of our sample). We display the temporal decay of the optical light curve and the time and the flux of the second data point observed in Table 4.

3. Optical light curves with plateaus at early times. The optical light curves of three bursts in our optical sample (GRBs 050525A, 060210, and 070411) display plateaus at early times. The plateaus in our optical sample are short, with the longest plateau lasting under $3500 \mathrm{~s}$. After the plateau ends, the light curves of these three bursts show a decay $\alpha_{O} \sim 1$, as expected for the external forward-shock emission. In Table 4, for these three bursts, we show the temporal decay after the plateau and the time and the flux of the second data point after the plateau.

4. Light curves with possible reverse shock emission at early times. Three GRBs in our optical sample (060111B, 060908, and 061126) show possible emission from the reverse shock. The light curves in this subgroup show an initial steep decline at early times, characteristic of the reverse shock, and then transition to a more shallow decay of $\alpha \sim 1$ that is more typical for the external-forward shock emission. For these GRBs, in Table 4 we show the temporal decay of the light curve and the time and flux of the second data point after the possible reverse shock emission.

\footnotetext{
13 The redshifts were taken from NASA's Swift GRB Table and Lookup website (exceptions are GRB 071003 (Perley et al. 2008) and GRBs 050502A and 071025 (J. Greiner's website)). Three GRBs (050721, 070420, 060111B) in our optical sample do not have a known redshift. For GRB 050721 and GRB 070420, we assumed the average redshift of 2.5 for Swift GRBs (Gehrels et al. 2009). The redshift for GRB 060111B was approximated as 2 in Stratta et al. (2009).
}

\subsection{Optical External-forward Shock Spectral Regime and Afterglow Parameter Assumptions}

When referring to the optical band, we will use $2 \mathrm{eV}$ since most of the light curves in our optical sample are either plotted at $2 \mathrm{eV}$ or were observed in the $R$ filter. As we did with the $\mathrm{X}$-ray sample, we will only consider the slow cooling ordering of the synchrotron characteristic frequencies, $v_{a}<v_{i}<v_{c}$. Because the optical light curve is declining at the time we are considering, the optical band must be above $v_{i}$ at this time. In Section 3.4, we argued that the X-ray band is between $v_{i}$ and $v_{c}$ at the EoSD at a few $\times 100 \mathrm{~s}$; therefore, the optical band must also be in this spectral regime at the early times $\left(\sim 10^{2}-10^{3} \mathrm{~s}\right)$ we are considering. The expression we will use to determine the optical external-forward shock flux is also Equation (7); however, we will have an equality (instead of an inequality), we replace $f_{1 \mathrm{keV} \text {,EoSD }}$ with $f_{2 \mathrm{eV}}$ (which represents the specific flux observed at $2 \mathrm{eV}$ ), and use $v_{14}$ corresponding to $2 \mathrm{eV}$.

The other afterglow parameters are determined as in Section 3.2: $\epsilon_{e}=0.2$ and with $z$ and the fluence, ${ }^{14}$ we obtain $E_{\text {iso }}^{\gamma}$ and use $E=5 E_{\text {iso. }}^{\gamma}$. As with our X-ray sample, we will display our $\epsilon_{B}$ results with $p=2.2,2.4$, and 2.8. We can also determine $p$ by using the temporal decay of the optical light curve, $\alpha_{O}$, which is shown in Table 4 for each burst (optical spectrum is not always available, so we cannot use the closure relations for the optical sample). In order to have $p>2$ for all of the bursts in our optical sample, we only consider a constant density medium when determining $p$ with $\alpha_{O}\left(\alpha_{O}=3(p-1) / 4\right.$ for $s=0$ and $\alpha_{O}=(3 p-1) / 4$ for $\left.s=2\right)$. Lastly, as we did for our X-ray sample, to keep the density dependence, we will plot the quantity $\epsilon_{B} n_{0}^{2 /(p+1)}\left(\epsilon_{B} A_{*-1}^{4 /(p+1)}\right)$ for $s=0(s=2)$.

\section{4. $\epsilon_{B}$ Results for Optical Sample}

We display the results for the measurements (from Equation (7)) on the quantity $\epsilon_{B} n_{0}^{2 /(p+1)}\left(\epsilon_{B} A_{*-1}^{4 /(p+1)}\right)$ for $s=0$ $(s=2)$ assuming that all the GRBs in our optical sample have $p=2.2,2.4$, and 2.8 in the top left, top right, and bottom left panels of Figure 3, respectively. Two histograms are shown in each panel, one for $s=0$ and the other for $s=2$. We also use $\alpha_{O}$ to determine $p$ (assuming $s=0$ ) and compare the results to the ones obtained with $p=2.4$ and $s=0$ (bottom right panel of Figure 3). In Table 3, we display a summary of the mean and median values of the measurements of the quantity $\epsilon_{B} n_{0}^{2 /(p+1)}\left(\epsilon_{B} A_{*-1}^{4 /(p+1)}\right)$ for $s=0(s=2)$ for each histogram. For the remainder of this section, we assume a standard $n_{0}=1$ $\left(A_{*-1}=1\right)$ for $s=0(s=2)$ when discussing our results for the $\epsilon_{B}$ measurements for our optical sample.

For a constant density (wind) medium, the mean and median $\epsilon_{B}$ measurements are $\sim$ few $\times 10^{-5}\left(\sim\right.$ few $\left.\times 10^{-6}\right)$. The mean and median $\epsilon_{B}$ measurements only change by a factor of a few when assuming a different value of $p$. To determine if assuming a standard $p=2.4$, as opposed to determining $p$ for each burst from $\alpha_{O}$, significantly affects the distribution of $\epsilon_{B}$ measurements, we compared the two histograms in the bottom right panel of Figure 3 with a Kolmogorov-Smirnov (K-S) test. The null hypothesis of the K-S test is that the two histograms are drawn from the same distribution. We test this null hypothesis at the 5\% significance level. The K-S test confirmed the null

\footnotetext{
${ }^{14}$ For the two bursts detected by INTEGRAL, the fluence in Table 4 is in the 20-200 keV band of the instrument IBIS (Imager on-Board the INTEGRAL Satellite, Ubertini et al. 2003): GRB 050502A (Gotz \& Mereghetti 2005) and GRB 080603A (Guidorzi et al. 2011).
} 
Table 4

Optical Sample Properties

\begin{tabular}{|c|c|c|c|c|c|c|c|c|c|}
\hline GRB & $z$ & $d_{L 28}$ & $\begin{array}{c}\text { Fluence } \\
\left(\times 10^{-6} \mathrm{erg} \mathrm{cm}^{-2}\right)\end{array}$ & $E_{\mathrm{iso}, 52}^{\gamma}$ & $\alpha_{O}$ & Ref. & $t_{2}$ & $\begin{array}{c}f_{2 \mathrm{eV}} \\
(\mathrm{mJy})\end{array}$ & $\begin{array}{c}\log _{10}\left(\epsilon_{B}\right) \\
(s=0)\end{array}$ \\
\hline 050401 & 2.9 & 7.67 & 8.22 & 15.55 & $0.80 \pm 0.03$ & [1] & 0.72 & 0.3 & -5.5 \\
\hline $050502 A$ & 3.793 & 10.59 & 1.4 & 4.12 & $1.16 \pm 0.03$ & [2] & 1 & 5 & -4.5 \\
\hline $050525 \mathrm{~A}$ & 0.606 & 1.10 & 15.3 & 1.45 & $1.12 \pm 0.05$ & [3] & 34.56 & 0.5 & -4.3 \\
\hline 050721 & 2.5 & 6.40 & 3.62 & 5.32 & $1.29 \pm 0.06$ & [4] & 20 & 0.2 & -5.0 \\
\hline 050730 & 3.97 & 11.19 & 2.38 & 7.53 & $0.89 \pm 0.05$ & [17] & 7.5 & 0.57 & -3.9 \\
\hline 050802 & 1.71 & 4.00 & 2.00 & 1.49 & $0.82 \pm 0.03$ & {$[1]$} & 3.6 & 0.5 & -3.5 \\
\hline 051111 & 1.55 & 3.54 & 4.08 & 2.52 & $1.00 \pm 0.02$ & [2] & 30 & 0.4 & -3.8 \\
\hline 051221A & 0.5465 & 0.97 & 1.15 & 0.09 & $0.96 \pm 0.03$ & [5] & 100 & 0.02 & -3.4 \\
\hline 060111B & 2 & 4.86 & 1.60 & 1.58 & $1.18 \pm 0.05$ & [6] & 2 & 0.4 & -5.2 \\
\hline 060210 & 3.91 & 10.99 & 7.66 & 23.65 & $1.03 \pm 0.06$ & [2] & 10 & 0.1 & -6.0 \\
\hline 060418 & 1.49 & 3.37 & 8.33 & 4.78 & $1.13 \pm 0.02$ & [7] & 2 & 8 & -4.6 \\
\hline 060607 A & 3.082 & 8.25 & 2.55 & 5.34 & $1.20 \pm 0.03$ & [7] & 2 & 10 & -4.2 \\
\hline 060904B & 0.703 & 1.32 & 1.62 & 0.21 & $1.00 \pm 0.18$ & [17] & 5.5 & 0.58 & -3.5 \\
\hline 060908 & 2.43 & 6.18 & 2.80 & 3.91 & $1.05_{-0.03}^{+0.03}$ & [8] & 2 & 2 & -4.5 \\
\hline 060927 & 5.6 & 16.81 & 1.13 & 6.08 & $1.21 \pm 0.06$ & [2] & 0.5 & 2 & -5.5 \\
\hline 061007 & 1.26 & 2.74 & 44.4 & 18.48 & $1.70 \pm 0.02$ & [7] & 2 & 50 & -6.0 \\
\hline 061110B & 3.44 & 9.42 & 1.33 & 3.34 & $1.64 \pm 0.08$ & [2] & 20 & 0.02 & -5.9 \\
\hline 061121 & 1.314 & 2.88 & 13.7 & 6.19 & $0.82 \pm 0.02$ & [7] & 4 & 0.5 & -4.7 \\
\hline 061126 & 1.159 & 2.47 & 6.77 & 2.39 & $0.89 \pm 0.02$ & [2] & 10 & 0.2 & -4.6 \\
\hline 070318 & 0.84 & 1.65 & 2.48 & 0.46 & $0.96 \pm 0.03$ & [7] & 20 & 0.2 & -3.7 \\
\hline 070411 & 2.954 & 7.84 & 2.70 & 5.27 & $0.92 \pm 0.04$ & [2] & 20 & 0.07 & -4.7 \\
\hline 070420 & 2.5 & 6.40 & 14.0 & 20.56 & $0.81 \pm 0.04$ & [2] & 3 & 0.8 & -4.7 \\
\hline 070714B & 0.92 & 1.85 & 0.72 & 0.16 & $0.83 \pm 0.04$ & [2] & 10 & 0.03 & -3.7 \\
\hline 071003 & 1.6 & 3.69 & 8.3 & 5.45 & $1.466 \pm 0.006$ & [9] & 0.6 & 20 & -5.7 \\
\hline 071025 & 5.2 & 15.41 & 6.5 & 31.26 & $1.27 \pm 0.04$ & [10] & 20 & 0.02 & -6.8 \\
\hline 071031 & 2.692 & 7.00 & 0.9 & 1.5 & $0.97 \pm 0.06$ & [11] & 10.5 & 0.4 & -3.4 \\
\hline $071112 \mathrm{C}$ & 0.823 & 1.61 & 3.00 & 0.53 & $0.95 \pm 0.02$ & {$[12]$} & 10.5 & 0.003 & -6.3 \\
\hline $080603 A$ & 1.688 & 3.94 & 1.1 & 0.80 & $0.99 \pm 0.07$ & {$[13]$} & 30 & 0.1 & -3.6 \\
\hline 080607 & 3.036 & 8.10 & 24.0 & 49.04 & 1.65 & {$[14]$} & 3 & 0.2 & -8.0 \\
\hline 080721 & 2.591 & 6.68 & 12.0 & 18.74 & $1.22 \pm 0.01$ & [5] & 3 & 10 & -5.0 \\
\hline 080810 & 3.35 & 9.13 & 4.60 & 11.06 & $1.23 \pm 0.01$ & [7] & 3 & 30 & -3.9 \\
\hline 080913 & 6.7 & 20.72 & 0.56 & 3.92 & $1.03 \pm 0.02$ & [15] & 10 & 0.02 & -5.2 \\
\hline 081008 & 1.967 & 4.76 & 4.30 & 4.13 & $0.96 \pm 0.03$ & [16] & 2 & 3 & -4.1 \\
\hline 090313 & 3.375 & 9.21 & 1.40 & 3.41 & $1.25 \pm 0.08$ & [17] & 20 & 2 & -3.4 \\
\hline 090418A & 1.608 & 3.71 & 4.60 & 3.05 & $1.21 \pm 0.04$ & [7] & 2 & 0.8 & -5.5 \\
\hline
\end{tabular}

Notes. The bursts in bold are also part of our X-ray sample, and the two bursts in italics were detected by INTEGRAL instead of Swift. The redshift and the corresponding luminosity distance in units of $10^{28} \mathrm{~cm}, d_{L 28}$, are shown in the second and third columns, respectively. The fluence, in units of $10^{-6} \mathrm{erg} \mathrm{cm}^{-2}$, is shown in the fourth column. In the next column we show the isotropic equivalent energy released in gamma rays during the prompt emission in the units of $10^{52} \mathrm{erg}, E_{\mathrm{iso}, 52}^{\gamma}$. The temporal decay of the optical light curve, $\alpha_{O}$, is shown in the sixth column. The reference where we found each optical light curve and $\alpha_{O}$ is shown in the seventh column. The time in units of $10^{2} \mathrm{~s}$ and the flux in mJy of the data point we used to determine $\epsilon_{B}$ are shown in the next two columns. For GRBs 050730 and 060904B, we display the time and flux at the peak of the optical light curve that are given in Melandri et al. (2010; we could not find an optical light curve in units of specific flux in the literature for these two bursts). In the last column we show the $\epsilon_{B}$ measurements for $n=1 \mathrm{~cm}^{-3}$ and $p$ determined from $\alpha_{O}$ (see bottom right panel of Figure 3).

References for light curves and $\alpha_{O}$. (1) Panaitescu et al. 2006; (2) Melandri et al. 2008; (3) Panaitescu 2007; (4) Antonelli et al. 2006; (5) Schulze et al. 2011; (6) Stratta et al. 2009; (7) Panaitescu \& Vestrand 2011; (8) Covino et al. 2010; (9) Perley et al. 2008; (10) Perley et al. 2009; (11) Krühler et al. 2009; (12) Uehara et al. 2010; (13) Guidorzi et al. 2011; (14) Perley et al. 2011; (15) Greiner et al. 2009; (16) Yuan et al. 2010; (17) Melandri et al. 2010.

hypothesis that the two histograms are consistent with being drawn from the same distribution.

As with the $\epsilon_{B}$ upper limits from X-ray data, the mean and median $\epsilon_{B}$ measurements decrease by about an order of magnitude when assuming a wind medium as opposed to a constant density medium. Compared to the distribution of $\epsilon_{B}$ upper limits we attained from X-ray data, the $\epsilon_{B}$ measurements from optical data show a much wider distribution. For a constant density (wind) medium, the $\epsilon_{B}$ measurements range from $\epsilon_{B} \sim 10^{-8}-10^{-3}\left(\epsilon_{B} \sim 10^{-9}-10^{-3}\right)$. Also, since we used the same equation (Equation (7)) to find both the upper limits on $\epsilon_{B}$ with X-ray data and the $\epsilon_{B}$ measurements with optical data, the discussion at the end of Section 3.5 on how the uncertainty in the afterglow parameters and the parameters $\xi$ and $f$ can affect the distribution of $\epsilon_{B}$ upper limits also applies to the distributions of $\epsilon_{B}$ measurements we presented in this section. In addition, since $\xi$ and $f$ are less than unity, including these two parameters will mean that our $\epsilon_{B}$ measurements are effectively upper limits on $\epsilon_{B}$.

\subsection{Comparison of Our Results on $\epsilon_{B}$ to Previous Studies}

We performed a K-S test between our optical $\epsilon_{B}$ measurements and the results from previous studies on $\epsilon_{B}$ (Figure 1). For our $\epsilon_{B}$ results, we used the optical $\epsilon_{B}$ measurements with $n=1 \mathrm{~cm}^{-3}$ and $p$ determined from $\alpha_{O}$ (filled-in histogram in 

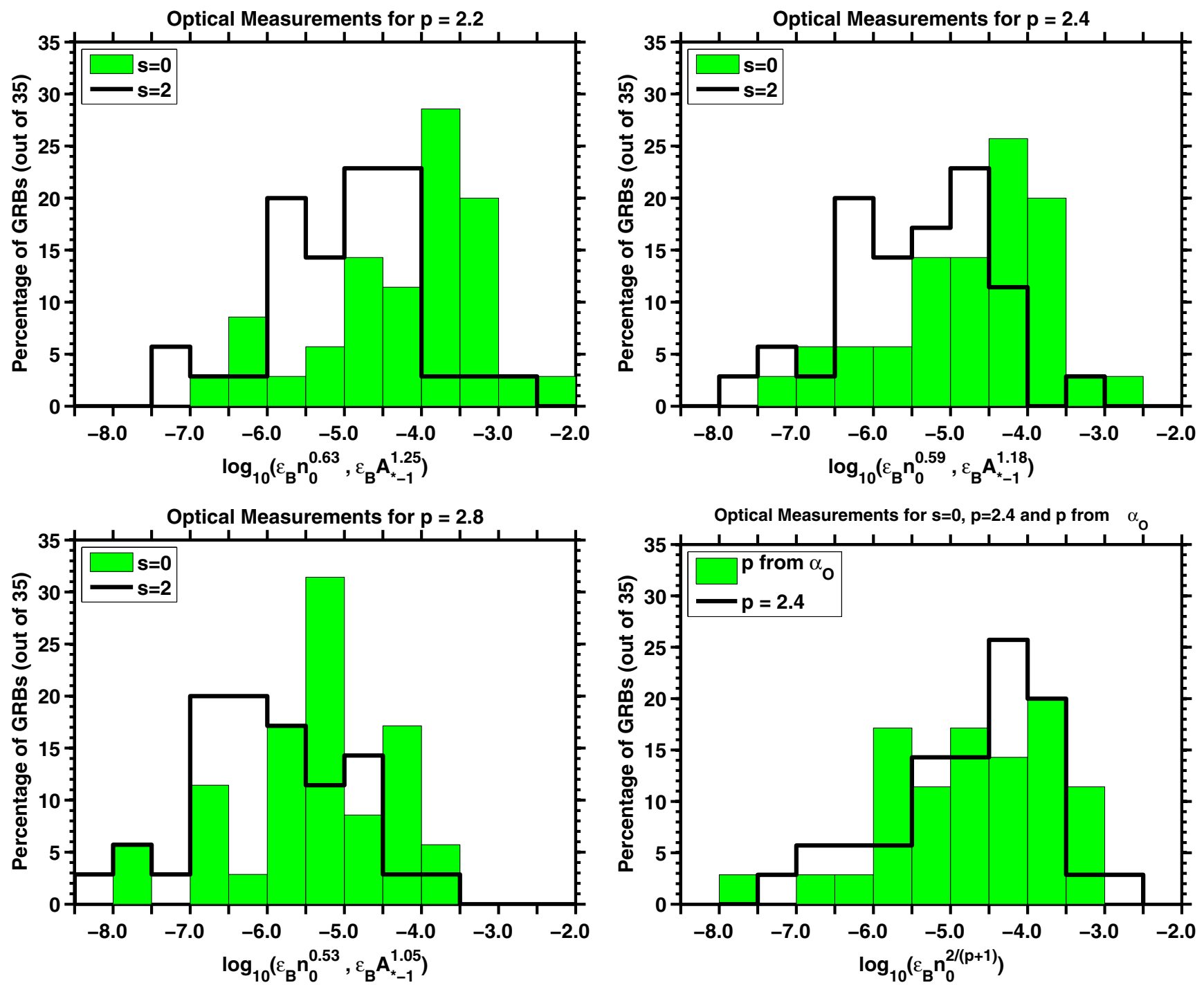

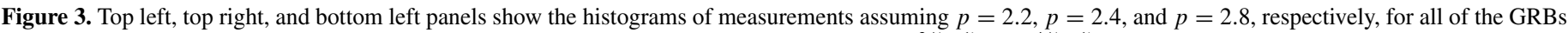

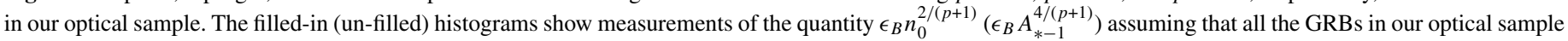

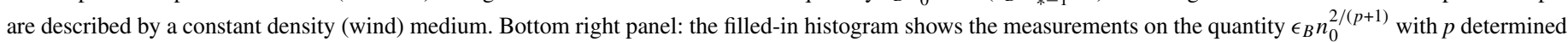

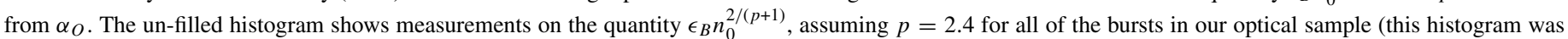
also shown in the top right panel).

(A color version of this figure is available in the online journal.)

the bottom right panel of Figure 3; the $\epsilon_{B}$ values are shown in Table 4). The result of the K-S test is that the null hypothesis is rejected. The $P$ value, which measures the probability that the null hypothesis is still true, is $2.1 \times 10^{-9}$. This result shows that the rejection of the null hypothesis is statistically significant. It is not surprising that the null hypothesis was rejected. The distribution from the previous studies is very inhomogeneous, with the values for $\epsilon_{B}$ being drawn from many different studies with different methodologies. Also, comparing the histogram in Figure 1 to the filled-in histogram in the bottom right panel of Figure 3, a couple of significant differences can be seen. The range for the histogram of $\epsilon_{B}$ values found in the literature is $\sim 10^{-5}-10^{-1}$, whereas the range for our $\epsilon_{B}$ results is $\sim 10^{-8}-10^{-3}$. The mean and median values for these two histograms are also significantly different. The mean (median) value for the $\epsilon_{B}$ histogram from the literature, $6.3 \times$ $10^{-2}\left(1.4 \times 10^{-2}\right)$, is a factor $\sim 700(\sim 600)$ times larger than the mean (median) $\epsilon_{B}$ value of the histogram with our results, which is $9.5 \times 10^{-5}\left(2.4 \times 10^{-5}\right)$.
One assumption that is commonly made in afterglow modeling studies is equipartition between $\epsilon_{e}$ and $\epsilon_{B}$. As we discussed in Section 2, the results for $\epsilon_{e}$ from the literature and the results from recent simulations of relativistic collisionless shocks support $\epsilon_{e} \sim 0.2$. From this result, many works assume $\epsilon_{B} \sim 10^{-2}-10^{-1}$. However, there is no physical argument to expect equipartition. Our distribution of $\epsilon_{B}$ upper limits and measurements, although wide, supports that there is no equipartition between electron and magnetic energies because none of the $\epsilon_{B}$ upper limits or measurements in our samples have a value larger than $\epsilon_{B} \sim$ few $\times 10^{-3}$. Another source of error that can lead to differences in $\epsilon_{B}$ values is differences in the determination of the spectral regime for the optical band. We took it to be between $v_{i}$ and $v_{c}$, but it is also possible for the optical band to be above $v_{c}$ at late times (e.g., Panaitescu \& Kumar 2002; Cenko et al. 2010). Another source for error is energy injection. We did not consider energy injection as a source of error because only $3 / 35$ of the bursts in our optical sample show plateaus (and these plateaus are short). The X-ray and optical light curves of many 
bursts show plateaus, and in these cases energy injection needs to be considered. Also, errors in our determination of fluxes and times from X-ray and optical light curves can also lead to small errors in $\epsilon_{B}$. In summary, the main assumption we made when determining $\epsilon_{B}$ is an efficiency of $\sim 20 \%$ in the conversion of kinetic energy to gamma-ray energy, and we did not assume equipartition between $\epsilon_{e}$ and $\epsilon_{B}$. Different authors have made different assumptions that can have a large effect on the results for $\epsilon_{B}$.

Lastly, for a few bursts, we checked whether our method of determining $\epsilon_{B}$ is consistent with the values determined for $\epsilon_{B}$ with other techniques. GRBs 980519 and 990123, discussed in the afterglow modeling study of Panaitescu \& Kumar (2002), have optical light curves that decline as a power law before the jet break. The optical band for both of these bursts was determined to be in the spectral regime $v_{i}<2 \mathrm{eV}<v_{c}$. Applying our technique to find a $\epsilon_{B}$ measurement for both of these bursts and using the value of $n$ reported in Panaitescu \& Kumar (2002) for both of these bursts, we find that these bursts have $\epsilon_{B} \sim 10^{-5}$, consistent with the results reported in Panaitescu \& Kumar (2002) for both of these bursts within a factor of a few. The small differences in $\epsilon_{B}$ values can be accounted for by differences in the coefficients used for the external-forward shock flux.

\section{GRBs IN COMMON TO BOTH OUR X-RAY AND OPTICAL SAMPLES}

A total of 14 bursts we considered are both in our X-ray and in our optical sample (GRB number is in bold in the first columns of Tables 1 and 4). In this section, we verify for these bursts that the X-ray $\epsilon_{B}$ upper limit is above the optical $\epsilon_{B}$ measurement. For the optical data, we will use the $\epsilon_{B}$ measurements with $p$ determined from $\alpha_{O}$ and $n=1 \mathrm{~cm}^{-3}$ (filled-in histogram in the bottom right panel of Figure 3). For the X-ray data, in this section, we will also consider $n=1 \mathrm{~cm}^{-3}(s=0)$, and we will use the value of $p$ determined from $\alpha_{O} \cdot{ }^{15}$ The comparison between the X-ray $\epsilon_{B}$ upper limits and the optical $\epsilon_{B}$ measurements is shown in Figure 4. This plot shows that all the X-ray $\epsilon_{B}$ upper limits are above the optical $\epsilon_{B}$ measurements.

\section{6. $E$ VERSUS $\epsilon_{B}$ CORRELATION?}

We now use the $\epsilon_{B}$ measurements from our optical sample to determine whether there is a correlation between $E$ and $\epsilon_{B}$. The three bursts studied in Kumar \& Barniol Duran (2009, 2010) have small values of $\epsilon_{B}$, consistent with shock compression of a seed magnetic field $B_{0} \sim$ few $\times 10 \mu \mathrm{G}$. One property that distinguishes these three GRBs is that they were particularly energetic, with $E_{\text {iso }}^{\gamma} \sim 10^{53}-10^{54} \mathrm{erg}$. Could the large energy intrinsic to these three bursts explain why they have low $\epsilon_{B}$ values? We investigate this possibility in Figure 5 by plotting the values of $E$ and $\epsilon_{B}$ for all the bursts in our optical sample.

For the energy of each burst, we assumed an efficiency of $\sim 20 \%$ ( $E=5 E_{\text {iso }}^{\gamma}$ ) in the conversion of the kinetic energy of the jet to gamma-ray radiation; the observables involved in calculating $E$ are the gamma-ray fluence and $z$. For the $\epsilon_{B}$ measurements, we used the values with $p$ determined from $\alpha_{O}$ and $n=1 \mathrm{~cm}^{-3}$. The observables involved in determining $\epsilon_{B}$

\footnotetext{
15 A total of 10 out of 14 of these bursts have optical data before $1000 \mathrm{~s}$. For these 10 bursts, we can use the optical data to check whether they satisfy the assumption we made in Section 3.1, $t_{\mathrm{dec}}<t_{\mathrm{EoSD}}$. Nine of these $10 \mathrm{GRBs}$ do satisfy this assumption; for the remaining GRB (080721), we are not able to check this assumption because the first optical observation (at $100 \mathrm{~s}$ ) is after the end of the steep decline $\left(t_{\mathrm{EoSD}}=70 \mathrm{~s}\right)$.
}

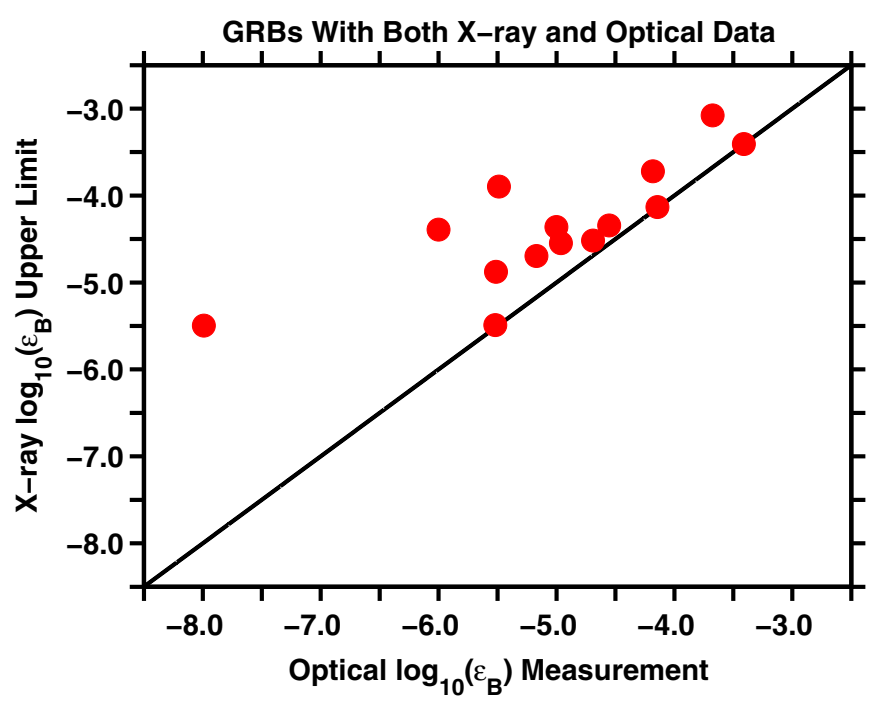

Figure 4. Comparison of the $\epsilon_{B}$ upper limits from X-ray data to the $\epsilon_{B}$ measurements from optical data. The 14 dots correspond to the 14 GRBs that are in both our X-ray and optical samples. The straight line indicates where the $\epsilon_{B}$ measurements are equal to the $\epsilon_{B}$ upper limits.

(A color version of this figure is available in the online journal.)

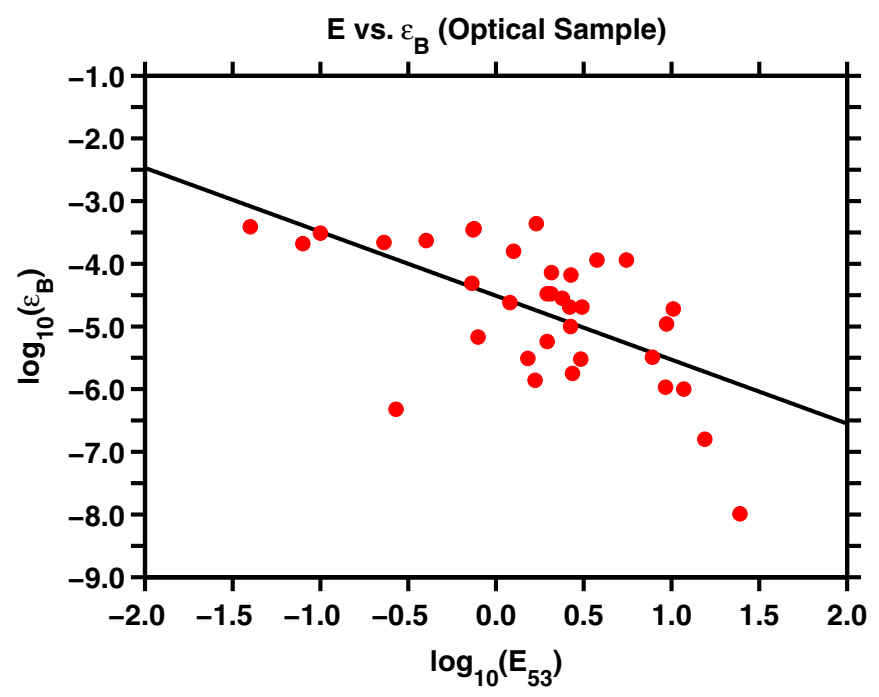

Figure 5. We plot the values of $E$ and the measurements of $\epsilon_{B}$ to determine whether they are correlated. The 35 points represent the GRBs in our optical sample, and the straight line is the best-fit line: $\log _{10}\left(\epsilon_{B}\right)=-1.02 \log _{10}\left(E_{53}\right)-$ 4.51 , with the slope of the line being $-1.02 \pm 0.23$ and the $y$-intercept of the line being $-4.51 \pm 0.16$. The correlation coefficient of the fit is 0.62 , and the $P$-value of the correlation is $1.2 \times 10^{-4}$ (3.8 $\sigma$ significance). The $\epsilon_{B}$ measurements are for $n=1 \mathrm{~cm}^{-3}$ and $p$ determined from $\alpha_{O}$ (shown in the filled-in histogram in the bottom right panel of Figure 3 and Table 4), and the values of $E$ were determined by assuming an efficiency $\sim 20 \%$ for all the GRBs in our optical samples.

(A color version of this figure is available in the online journal.)

are the observed specific optical flux and the time. In Figure 5, we also show the best-fit line. ${ }^{16}$ This fit shows that an increase

\footnotetext{
16 From Equation (7), it may be expected that $E$ and $\epsilon_{B}$ are correlated. This is not necessarily true because each burst has a different value for the observed quantities $f_{v}$ and $t$. In addition, since $p$ was determined from $\alpha_{O}$, each burst has a different $p$. We also checked whether $E$ and $\epsilon_{B}$ were correlated when assuming a fixed $p=2.4$. From Equation (9), we would expect the slope of the line to be -1.59 . The best-fitting line for $p=2.4$ is $\log _{10}\left(\epsilon_{B}\right)=$ $-0.77 \log _{10}\left(E_{53}\right)-4.53$, with the slope of the line being $-0.77 \pm 0.24$ and the $y$-intercept being $-4.53 \pm 0.16$. The slope of this line is more than $3 \sigma$ away from -1.59 , showing that there is no expected correlation between $E$ and $\epsilon_{B}$ for our methodology of determining $\epsilon_{B}$.
} 
in $E$ leads to a decrease in $\epsilon_{B}$. The correlation coefficient of the fit is 0.62 , indicating that there is a weak correlation between $E$ and $\epsilon_{B}$. A value of the correlation coefficient close to 1 would indicate a strong correlation. The $P$ value of the correlation is $1.2 \times 10^{-4}$ (3.8 $\sigma$ significance), indicating that there is a small probability that the correlation occurred by chance.

Although many points show large deviations from the bestfit line, the scatter of the points may be reduced or increased by the uncertainty in the afterglow parameters. An error in the efficiency would affect the values of $E$, and an error in $\epsilon_{e}$, the efficiency, or $n$ would affect the $\epsilon_{B}$ measurements (see Section 3.5 for a discussion on how the $\epsilon_{B}$ measurements would be affected when an error in an afterglow parameter is made). It is possible that the uncertainty in $E$ and $\epsilon_{B}$ can reduce or increase the scatter and make the correlation between $E$ and $\epsilon_{B}$ stronger or weaker.

\section{MAGNETIC FIELD AMPLIFICATION FACTOR FOR X-RAY AND OPTICAL RESULTS}

In Sections 3-5, we presented our results for the strength of the magnetic field downstream of the shock front in terms of the afterglow parameter $\epsilon_{B}$. If shock compression was the only mechanism amplifying the ambient magnetic field (assuming a standard $B_{0} \sim$ few $\mu \mathrm{G}$ and a standard $n=1 \mathrm{~cm}^{-3}$ ), then $\epsilon_{B} \sim 10^{-9}$ is expected. Most of the bursts in our distributions of $\epsilon_{B}$ upper limits and measurements have values larger than $\epsilon_{B} \sim$ $10^{-9}$. These results suggest that amplification of the magnetic field, in addition to shock compression, is needed to explain the afterglow observations. In this section, we will present our results in terms of an amplification factor, which quantifies the amplification that is needed, beyond shock compression, to explain the observations.

If shock compression were the only mechanism amplifying the seed magnetic field $B_{0}$, then $B=4 \Gamma B_{0}$. To quantify how much additional amplification of the ambient magnetic field is needed, beyond shock compression, we define the amplification factor, $\mathrm{AF}$, as

$$
\mathrm{AF} \equiv \frac{B}{4 B_{0} \Gamma} .
$$

$\mathrm{AF}$ is a constant that satisfies $\mathrm{AF} \geqslant 1$ since $B \geqslant 4 B_{0} \Gamma$. $\mathrm{AF}=1$ means that the observed $B$ is consistent with the only amplification arising from seed magnetic field shock compression. The expression for $\epsilon_{B}$ is $\epsilon_{B}=B^{2} / 32 \pi m_{p} c^{2} n \Gamma^{2}$. With the definition for AF, $\epsilon_{B}$ is

$$
\epsilon_{B}=(\mathrm{AF})^{2} \times \frac{B_{0}^{2}}{2 \pi n m_{p} c^{2}} .
$$

We note that $\epsilon_{B}$ is given by (AF) ${ }^{2}$ times the $\epsilon_{B}$ we would get if shock compression were the only mechanism amplifying the magnetic field.

We will now use Equation (13) and our previous results for the X-ray $\epsilon_{B}$ upper limits to determine an upper limit on AF. In Section 3.5, if we assumed a standard $n=1 \mathrm{~cm}^{-3}$, we were able to attain an upper limit on $\epsilon_{B}$ for a constant density medium. ${ }^{17}$ We will refer to these $\epsilon_{B}$ upper limits as $\overline{\epsilon_{B}}\left(p, n_{0}=1\right)$. In the notation $\overline{\epsilon_{B}}\left(p, n_{0}=1\right)$, the bar over $\epsilon_{B}$ signifies that this is an upper limit on $\epsilon_{B}$, the $p$ in the parentheses shows that the $\epsilon_{B}$ upper limit depends on the value of $p$ we used, and the

\footnotetext{
17 We will only consider a constant density medium when displaying the results for the amplification factor. We will show in Equation (15) that AF has a weak dependence on the density.
}

Table 5

Mean and Median AF Values for the X-Ray (Upper Limits on AF) and Optical (Measurements of AF) Histograms Shown in Figures 6 and 7

\begin{tabular}{lcccc}
\hline \hline $\begin{array}{l}\text { X-ray } \\
(s=0)\end{array}$ & $p=2.2$ & $p=2.4$ & $p=2.8$ & \\
\hline Mean & 84 & 67 & 62 & \\
Median & 63 & 51 & 44 & \\
\hline Opt. & & & & \\
$(s=0)$ & $p=2.2$ & $p=2.4$ & $p=2.8$ & $p$ from $\alpha_{\mathrm{O}}$ \\
\hline Mean & 130 & 71 & 36 & 71 \\
Median & 100 & 56 & 23 & 48 \\
\hline
\end{tabular}

Notes. All the labels are the same as in Table 3 . A constant density medium with $n=1 \mathrm{~cm}^{-3}$ (the amplification factor has a weak dependence on the density; see Section 7 and Equation (15)) and a seed magnetic field $B_{0}=10 \mu \mathrm{G}$ were assumed for all the bursts in our X-ray and optical samples.

$n_{0}=1$ shows that we assumed $n=1 \mathrm{~cm}^{-3}$. With this notation, we can keep the dependence of the $\epsilon_{B}$ upper limit on $n$ (see Equation (7)):

$$
\epsilon_{B}<\frac{\overline{\epsilon_{B}}\left(p, n_{0}=1\right)}{n_{0}^{2 /(p+1)}} .
$$

Combining Equations (13) and (14), the expression for the upper limit on AF is

$$
\mathrm{AF}<\frac{1.0 \times 10^{4}\left[\overline{\epsilon_{B}}\left(p, n_{0}=1\right)\right]^{1 / 2}}{B_{0,10 \mu \mathrm{G}} n_{0}^{(1-p) /(2 p+2)}},
$$

where $B_{0,10 \mu \mathrm{G}} \equiv B_{0} / 10 \mu \mathrm{G}$. One advantage to expressing the results of the magnetic field downstream of the shock front in terms of AF is that AF depends weakly on $n$. For a standard $p=2.4$, AF $\propto n^{0.21}$. On the other hand, there is a strong dependence on $B_{0}, \mathrm{AF} \propto B_{0}^{-1}$.

\subsection{Amplification Factor Upper Limit for Our X-Ray Sample}

We will now show the results for the AF upper limits we obtained from Equation (15). Since the amplification factor has a weak dependence on the density, we will assume a standard $n_{0}=1$ when displaying the results for the AF upper limits. When plotting the results for $\mathrm{AF}$, we will keep the dependence on $B_{0}$ and plot the quantity $(\mathrm{AF}) B_{0,10 \mu \mathrm{G}}$. In the left and right panels of Figure 6, we show the upper limits on the quantity (AF) $B_{0,10 \mu \mathrm{G}}$ for a fixed $p=2.4$ and $p=2.2,2.8$, where the values of $\overline{\epsilon_{B}}\left(p, n_{0}=1\right)$ used in Equation (15) were shown in top right, top left, and bottom panels of Figure 2, respectively. For the remainder of this section, we will assume $B_{0}=10 \mu \mathrm{G}$ when discussing the results for the AF upper limits.

The mean and median values of the AF upper limits are summarized in Table 5. The mean (median) AF upper limits are in the range $\mathrm{AF} \sim 60-80$ (AF $\sim 40-60)$. The AF upper limit histograms show a wide distribution, with a range of $\sim 10$ to $\sim 300$. To determine if assuming a different value of $p$ has a significant effect on the distribution of AF upper limits, we performed a K-S test between the histograms in the right panel of Figure 6. The K-S test confirmed the null hypothesis, leading us to conclude that the AF upper limit results are not sensitive to the value of $p$ we assume.

We now discuss how an error in each of the afterglow parameters can affect our results for the AF upper limits. For this discussion, we will assume $p=2.4$. From Equation (15), $\mathrm{AF} \propto \sqrt{\overline{\epsilon_{B}}\left(p=2.4, n_{0}=1\right)} B_{0}^{-1} n^{0.2}$, and from Equation (9), 

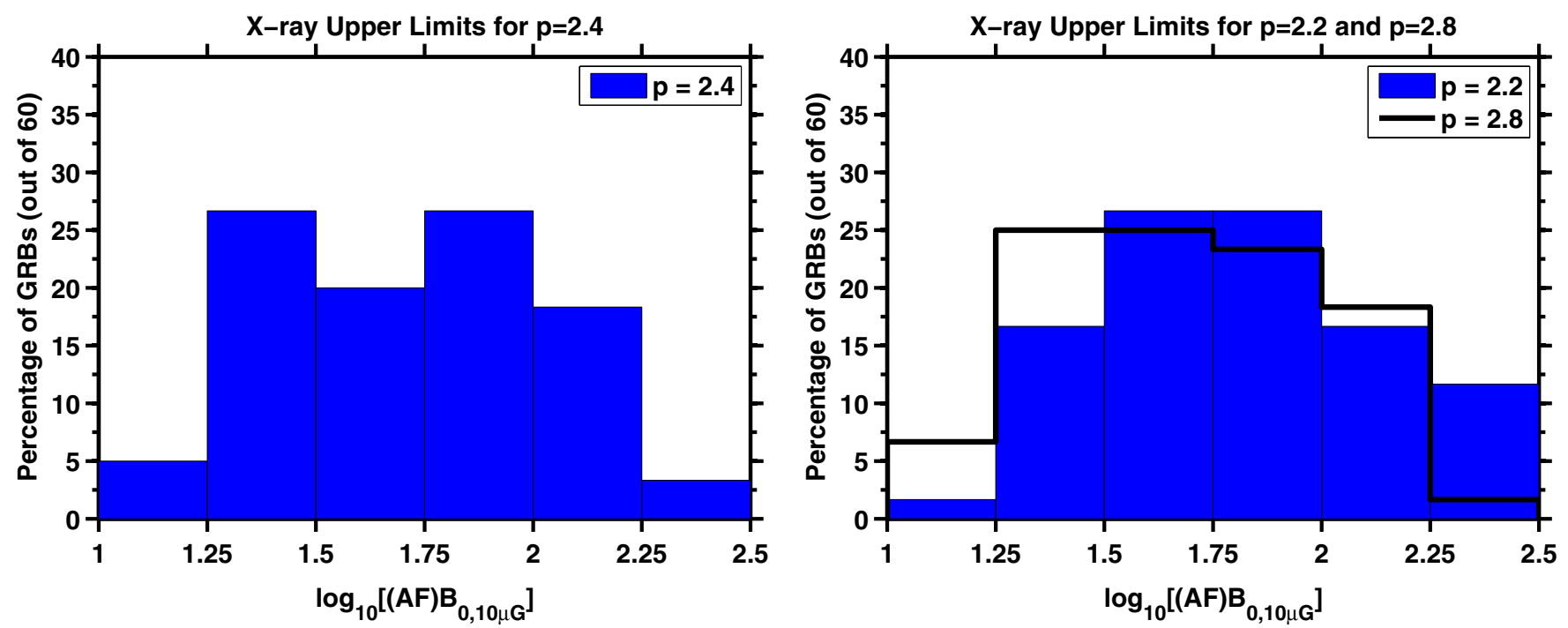

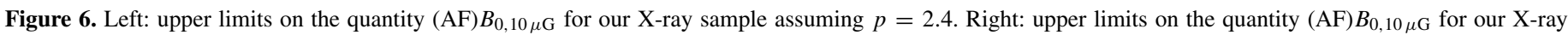

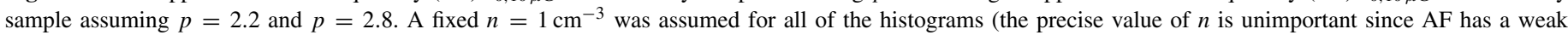
dependence on $n$; see Section 7 and Equation (15)).

(A color version of this figure is available in the online journal.)

$\epsilon_{B} \propto \epsilon_{e}^{-1.6} E^{-1.6} n^{-0.6}$. From these two expressions, AF $\propto$ $\epsilon_{e}^{-0.8} E^{-0.8} n^{0.2} B_{0}^{-1}$. We note that compared to the $\epsilon_{B}$ upper limit (Equation (9)), the AF upper limit has a weaker dependence on $\epsilon_{e}, E$, and $n$. As we discussed at the end of Section 3.5, a likely error in $\epsilon_{e}$ is a factor of $\sim 2$; this error in $\epsilon_{e}$ will translate into an error in the AF upper limits by a factor of only $\sim 2$. For the energy, we assumed an efficiency $\sim 20 \%$, and a likely error in the efficiency is a factor $\sim 2-3$ (see Section 3.5); this error in the efficiency would lead to an error in the AF upper limits by only a factor $\sim 2$. One advantage to expressing the results of the magnetic field downstream of the shock front in terms of AF is that $\mathrm{AF}$ has a very weak dependence on $n$. An error in $n$ by a factor $\sim 10^{3}$ (see Section 3.5) from our assumed $n=1 \mathrm{~cm}^{-3}$ will only lead to an error in the AF upper limits by a factor $\sim 4$. The price to pay for a weak $n$ dependence is a linear dependence on $B_{0}$, with $\mathrm{AF} \propto B_{0}^{-1} . B_{0}$ is an uncertain parameter that likely varies from GRB environment to GRB environment, and it is the largest source of uncertainty for AF.

\subsection{Amplification Factor Measurement for Our Optical Sample}

As we discussed in Section 4.2, for our optical sample, we found a measurement for $\epsilon_{B}$ instead of an upper limit. This will allow us to determine a measurement for AF. To do this, we will use Equation (15), but in this case we have an equality instead and we have $\epsilon_{B}\left(p, n_{0}=1\right)$ instead of $\overline{\epsilon_{B}}\left(p, n_{0}=1\right)$. The notation $\epsilon_{B}\left(p, n_{0}=1\right)$ denotes the $\epsilon_{B}$ measurements for our optical sample from Section 4.4 if we assume a standard $n=1 \mathrm{~cm}^{-3}$. Also, as with the X-ray sample, we only consider $s=0$ when calculating the AF measurements and assume a fixed $n=1 \mathrm{~cm}^{-3}$. In the left panel of Figure 7, we show the results for the measurements on the quantity (AF) $B_{0,10 \mu \mathrm{G}}$ for $p$ determined from $\alpha_{O}$ and also assuming $p=2.4$. In the right panel of Figure 7, we show the measurements on the quantity (AF) $B_{0,10 \mu \mathrm{G}}$ for $p=2.2$ and $p=2.8$. For the remainder of this section, we will assume $B_{0}=10 \mu \mathrm{G}$ when discussing the results for the AF measurements.

A summary of the mean and median AF measurements for our optical sample is shown in Table 5. To determine whether assuming a standard $p=2.4$, as opposed to determining $p$ from $\alpha_{O}$ for each burst, has a statistically significant effect on the distribution of AF measurements, we performed a K-S test between the two histograms in the left panel of Figure 7. The $\mathrm{K}-\mathrm{S}$ test confirmed the null hypothesis. The mean (median) AF measurements for the optical histograms range from $\sim 40$ to $\sim 130(\sim 20$ to $\sim 100)$. Compared to the AF upper limit histograms, the AF measurement histograms show a wider distribution, ranging from $\mathrm{AF} \sim 1$ to $\mathrm{AF} \sim 1000$. Also, since we used the same expression to determine the AF upper limits and measurements (Equation (15)), the discussion at the end of Section 7.1 on how an error in one of the afterglow parameters can affect the AF upper limits also applies to the AF measurements. ${ }^{18}$

\section{DISCUSSION AND CONCLUSIONS}

In this work, we presented a systematic study on the magnetic field downstream of the shock front for large samples of GRBs (60 in our X-ray sample and 35 in our optical sample). We expressed the strength of the downstream magnetic field in terms of both the afterglow parameter $\epsilon_{B}$ and an amplification factor, denoted by $\mathrm{AF}$, which quantifies how much amplification, beyond shock compression of the seed magnetic field, is needed to explain the downstream magnetic field. This is the first time a large and systematic study has been carried out to study $\epsilon_{B}$ and to determine how much amplification of the seed magnetic field is required by the observations. For our X-ray (optical) sample, we determined an upper limit (measurement) for both $\epsilon_{B}$ and AF. The upper limits on $\epsilon_{B}$ and AF for our X-ray sample

\footnotetext{
$18 \xi$ and $f$ also affect our AF results. To account for $\xi$, since the $\epsilon_{B}$ upper limit/measurement is $\propto \xi^{4(p-2) /(p+1)}$ and the AF upper limit/measurement is $\propto\left(\overline{\epsilon_{B}}\left(p, n_{0}=1\right)\right)^{1 / 2}, \mathrm{AF} \propto \xi^{2(p-2) /(p+1)}$. To account for $f$, since $\mathrm{AF} \propto\left(\overline{\epsilon_{B}}\left(p, n_{0}=1\right)\right)^{1 / 2}, \mathrm{AF} \propto f^{1 / 2}$. Thus, including $\xi$ and $f$ will decrease the values of the AF upper limits/measurements. Taking the lowest possible value for $f$, the AF upper limits/measurements can decrease by up to a factor $\sim 40$. This would make $\sim 50 \%(\sim 60 \%)$ of the bursts in our X-ray (optical) sample consistent with shock compression. In addition, as with the $\epsilon_{B}$ measurements, since $\xi$ and $f$ are less than unity, including these two parameters will mean that our AF measurements will become upper limits on AF.
} 

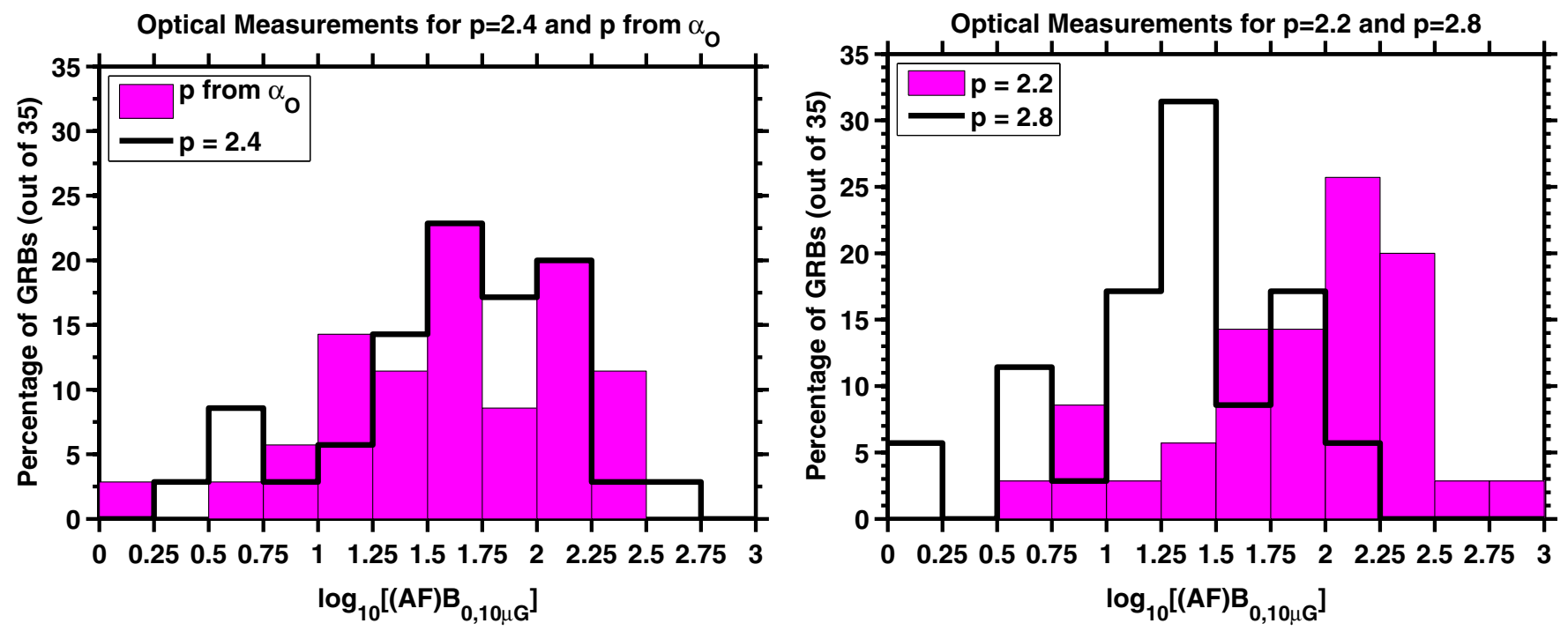

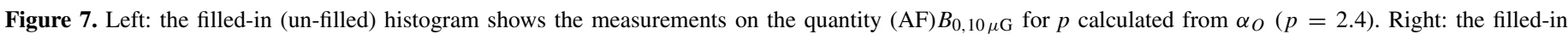

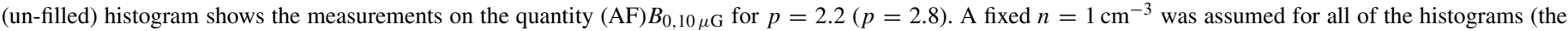
precise value of $n$ is unimportant since AF has a weak dependence on $n$; see Section 7 and Equation (15)).

(A color version of this figure is available in the online journal.)

were found from the constraint that the observed flux at the EoSD is greater than or equal to the external-forward shock flux. This is a new method to constrain $\epsilon_{B}$ that relies on the steep decline emission, which has been observed by Swift for many GRBs. Our optical sample was restricted to light curves that decline with $\alpha \sim 1$ from the early times $\sim 10^{2}-10^{3} \mathrm{~s}$, as expected for the external-forward shock emission. We found the measurements for $\epsilon_{B}$ and $\mathrm{AF}$ for our optical sample from the condition that the observed flux is equal to the external-forward shock flux.

The condition used for our X-ray (optical) sample was converted into an upper limit (measurement) on the quantity $\epsilon_{B} n^{2 /(p+1)}$ for $s=0$ or $\epsilon_{B} A_{*-1}^{4 /(p+1)}$ for $s=2$ by assuming a $\sim 20 \%$ efficiency in the conversion of kinetic energy to prompt gamma-ray radiation. To find an upper limit (measurement) on $\epsilon_{B}$ for our X-ray (optical) sample, we assumed a standard $n=1 \mathrm{~cm}^{-3}\left(A_{*}=0.1\right)$ for the density for a constant density (wind) medium. A discussion on how the uncertainty in the afterglow parameters affects our results for $\epsilon_{B}$ can be found at the end of Section 3.5. The largest source of uncertainty for our results on $\epsilon_{B}$ is the density, since the value of the density has been observed to vary over many orders of magnitude and its precise value is not known for each GRB (see Section 3.2). For the bursts that are in both our X-ray and optical samples, we also applied a consistency check to make sure our results for $\epsilon_{B}$ are correct (see Section 5).

From Table 3, for a constant density (wind) medium, most of the $\epsilon_{B}$ upper limit and measurement histograms have a median value $\sim$ few $\times 10^{-5}\left(\sim\right.$ few $\left.\times 10^{-6}\right)$. These results imply that half of the bursts in both our X-ray and optical samples have an $\epsilon_{B}$ value $\sim$ few $\times 10^{-5}$ or lower. Assuming $n=1 \mathrm{~cm}^{-3}$ and $B_{0} \sim$ few $\times \mu \mathrm{G}$, shock compression is only able to produce $\epsilon_{B} \sim 10^{-9}$. Although $\epsilon_{B} \sim 10^{-9}$ is 4 orders of magnitude lower than $\epsilon_{B} \sim 10^{-5}, \epsilon_{B} \sim 10^{-5}$ is smaller by $\sim 2-4$ orders of magnitude compared to the majority of previously reported $\epsilon_{B}$ values (Figure 1), which are $\epsilon_{B} \sim 10^{-3}-10^{-1}$. Assuming $B_{0} \sim 10 \mu \mathrm{G}, \epsilon_{B} \sim$ few $\times 10^{-5}$ corresponds to $\mathrm{AF} \sim 50$ (Equation (15)). Our result of a median $\epsilon_{B} \sim$ few $\times 10^{-5}$ shows that the majority of the bursts in our X-ray and optical samples only require a weak amplification beyond shock compression, by a factor $\sim 50$ or lower.

The near equipartition $\epsilon_{B} \sim 0.01-0.1$ determined near the shock front by theoretical studies and particle-in-cell (PIC) simulations (Medvedev \& Loeb 1999; Chang et al. 2008; Martins et al. 2009; Keshet et al. 2009; Lemoine 2013; Sironi et al. 2013) stands in contrast with our median results of $\epsilon_{B} \sim$ few $\times 10^{-5}$. PIC simulations of relativistic collisionless shocks performed by Chang et al. (2008) and Keshet et al. (2009) found that the magnetic field generated near the shock front decays with distance downstream of the shock front. Lemoine (2013) and Lemoine et al. (2013) studied the effects that this decaying magnetic field has on the shock accelerated electrons radiating afterglow emission downstream of the shock front. The main effect is that electrons with different Lorentz factors cool in regions with different magnetic fields, with the higher (lower) energy photons being emitted by electrons that are closer to (farther from) the shock front (Lemoine et al. 2013). Considering the decay of the downstream magnetic field, Lemoine et al. (2013) modeled the afterglow data of four GRBs that have extended emission at energies $>100 \mathrm{MeV}$ (detected by Fermi/ LAT) and also X-ray, optical, and radio data. Their afterglow modeling results for the X-ray, optical, and radio data found $\epsilon_{B} \sim 10^{-6}-10^{-4}$, consistent with our results for the median $\epsilon_{B}$ upper limits and measurements attained from X-ray and optical data.

Our next main result relates to the distribution of $\epsilon_{B}$ values. One property the $\epsilon_{B}$ values from the literature shared with our optical $\epsilon_{B}$ measurements is that they both show a wide distribution. The literature compilation (Figure 1) showed $\epsilon_{B} \sim$ $10^{-5}-10^{-1}$, and our optical $\epsilon_{B}$ measurement histograms showed an even wider distribution, in the range $\epsilon_{B} \sim 10^{-8}-10^{-3}$ $\left(\epsilon_{B} \sim 10^{-9}-10^{-3}\right)$ for a constant density (wind) medium. One possibility we investigated to explain the wide distribution of $\epsilon_{B}$ values is whether bursts with smaller $\epsilon_{B}$ values are more energetic than bursts with larger $\epsilon_{B}$ values (Figure 5). Although the bursts in our optical sample did show the trend that bursts 
with larger $E$ have a smaller $\epsilon_{B}$, the correlation was weak, with a correlation coefficient of 0.62 and a $P$ value of $1.2 \times 10^{-4}$ (3.8 $\sigma$ significance).

Another possibility to explain the wide distribution of $\epsilon_{B}$ relates to the uncertainty in the environmental parameters $\left(B_{0}\right.$ and $n$ ) in the medium surrounding GRBs. One possibility to explain bursts with values of $\epsilon_{B} \sim 10^{-5}-10^{-3}$, under the interpretation of weak amplification beyond shock compression, is that they occurred in environments with particularly high seed magnetic fields. Since $\epsilon_{B} \propto B_{0}^{2}$, an increase in $B_{0}$ by an order of magnitude will lead to an increase in $\epsilon_{B}$ by two orders of magnitude. A weak amplification beyond shock compression of $\mathrm{AF} \sim 50$ (as inferred for the bursts with $\epsilon_{B} \sim$ few $\times 10^{-5}$ ) and a strong $B_{0} \sim 10 \mu \mathrm{G}-100 \mu \mathrm{G}$ yields $\epsilon_{B} \sim 10^{-5}-10^{-3}$ (see Equation (15)). We note that for this estimate we assumed $n=1 \mathrm{~cm}^{-3}$, but as we discussed in Section 7 , the amplification factor has a weak dependence on the density. This possibility, that the larger values of $\epsilon_{B}$ may be explained by bursts going off in environments with particularly strong $B_{0}$, will be discussed further in Barniol Duran (2013).

In addition to many bursts in our optical sample having particularly large $\epsilon_{B} \sim 10^{-5}-10^{-3}$, there were also some bursts in our optical sample that have particularly low $\epsilon_{B}$ values. For $p$ determined from $\alpha_{O}$ and $n=1 \mathrm{~cm}^{-3}$ (bottom right panel of Figure 3), GRBs 071025 and $071112 \mathrm{C}$ have $\epsilon_{B} \sim 10^{-7}$ and GRB 080607 has $\epsilon_{B} \sim 10^{-8}$. Assuming $n=1 \mathrm{~cm}^{-3}$ and $B_{0}=10 \mu \mathrm{G}$, these bursts with $\epsilon_{B} \sim 10^{-8}-10^{-7}$ are consistent with shock compression of a seed magnetic field of a few $10 \mu \mathrm{G}$ being the only amplification necessary to explain the observations.

Lastly, we mention that a similar conclusion for radio observations of supernova remnants (SNRs) was reached in Thompson et al. (2009). Like GRB afterglow emission, SNR emission arises from a blast wave interacting with the surrounding medium, but at non-relativistic speeds. Thompson et al. (2009) found that starburst galaxies have strong ambient magnetic fields $\sim 1 \mathrm{mG}$, and they concluded that shock compression of this strong seed magnetic field is enough to explain the radio emission from SNRs. On the other hand, for normal spiral galaxies with ambient magnetic fields $\sim 5-10 \mu \mathrm{G}$, they concluded that additional amplification beyond shock compression by a factor $\sim 3-7$ was necessary to explain the radio emission from SNRs.

This work made use of data supplied by the UK Swift Science Data Centre at the University of Leicester. R.S. dedicates this work to his dear friend, Jossue Colato, thanks the McDonald Observatory for supporting this research, Patrick Crumley and Roberto Hernández for helpful discussions, Kevin Gately for his help with obtaining the values of $\epsilon_{e}$ and $\epsilon_{B}$ from the literature, and the referee for reviewing the manuscript. This work has been funded in part by NSF grant ast-0909110. R.B.D. was supported by an ERC advanced grant (GRB) and by the I-CORE Program of the PBC and the ISF (grant 1829/12).

\section{APPENDIX}

\section{$\epsilon_{e}$ AND $\epsilon_{B}$ VALUES FROM THE LITERATURE}

In this Appendix, we show a table of the $\epsilon_{e}$ and $\epsilon_{B}$ values we used to make the histograms in Figure 1. For the first GRBs with high-quality afterglow data, different works (e.g., Wijers \& Galama 1999; Chevalier \& Li 1999, 2000; Li \& Chevalier 2001; Harrison et al. 2001; Yost et al. 2003) have presented
Table 6

$\epsilon_{e}$ and $\epsilon_{B}$ Values from the Literature

\begin{tabular}{|c|c|c|c|}
\hline GRB & $\epsilon_{e}$ & $\epsilon_{B}$ & Ref. \\
\hline 970508 & 0.62 & 0.10 & [1] \\
\hline 980329 & 0.12 & 0.17 & {$[2]$} \\
\hline 980519 & 0.25 & $\left(3.5_{-2.3}^{+32}\right) \times 10^{-5}$ & [1] \\
\hline 980703 & 0.14 & $4.6 \times 10^{-4}$ & [3] \\
\hline 990123 & 0.59 & $\left(7.4_{-5.9}^{+23}\right) \times 10^{-4}$ & [1] \\
\hline 990510 & $>0.3$ & $6 \times 10^{-3}$ & [4] \\
\hline 991208 & 0.32 & $2.1 \times 10^{-2}$ & [1] \\
\hline 991216 & 0.4 & $2 \times 10^{-2}$ & [4] \\
\hline $000301 \mathrm{C}$ & 0.4 & $7 \times 10^{-2}$ & [4] \\
\hline 000926 & 0.35 & $\left(6.5_{-1.1}^{+1.5}\right) \times 10^{-2}$ & [1] \\
\hline 010222 & $>0.3$ & $2 \times 10^{-4}$ & [4] \\
\hline 011211 & 0.22 & $5.0 \times 10^{-4}$ & {$[5]$} \\
\hline 020405 & 0.1 & 0.3 & [6] \\
\hline 020813 & & $4.0 \times 10^{-4}$ & [5] \\
\hline 021004 & 0.21 & $2 \times 10^{-4}$ & [7] \\
\hline 030226 & 0.11 & $2.5 \times 10^{-4}$ & [5] \\
\hline 030329 & 0.16 & 0.10 & [8] \\
\hline 050416A & $0.2-0.333$ & $0.072-0.333$ & [9] \\
\hline 050820A & $0.14_{-0.01}^{+0.02}$ & $0.013_{-0.001}^{+0.003}$ & [10] \\
\hline 050904 & 0.02 & 0.015 & [11] \\
\hline 051022 & $0.0681_{-0.0348}^{+0.3951}$ & $\left(8.02_{-7.17}^{+28.18}\right) \times 10^{-3}$ & {$[12]$} \\
\hline 051221A & $0.24-0.333$ & $0.12-0.333$ & {$[13]$} \\
\hline 060418 & $0.06_{-0.02}^{+0.01}$ & $0.15_{-0.01}^{+0.14}$ & {$[10]$} \\
\hline 070125 & $0.27_{-0.01}^{+0.03}$ & $0.0277_{-0.0075}^{+0.0044}$ & {$[14]$} \\
\hline 080129 & 0.4 & $5 \times 10^{-2}$ & {$[15]$} \\
\hline 080319B & $0.11 \pm 0.01$ & 0.33 & {$[10]$} \\
\hline 080928 & 0.165 & $\left(2.5_{-2.4}^{+16}\right) \times 10^{-4}$ & {$[16]$} \\
\hline 090323 & $0.070_{-0.005}^{+0.005}$ & $0.0089_{-0.0018}^{+0.0007}$ & {$[17]$} \\
\hline 090328 & $0.11_{-0.01}^{+0.06}$ & $0.0019_{-0.0008}^{+0.0004}$ & {$[17]$} \\
\hline 090423 & 0.28 & $1.6 \times 10^{-4}$ & [18] \\
\hline
\end{tabular}

Notes. In this table, we show all of the $\epsilon_{e}$ and $\epsilon_{B}$ values, determined in previous afterglow modeling studies, that we were able to find in the literature. These values are plotted in the histograms in Figure 1. In the column labeled Ref. we give the reference where we found each value of $\epsilon_{e}$ and $\epsilon_{B}$.

References. (1) Panaitescu \& Kumar 2002; (2) Yost et al. 2002; (3) Panaitescu \& Kumar 2001b; (4) Panaitescu \& Kumar 2001a; (5) Panaitescu 2005; (6) Berger et al. 2003b; (7) Björnsson et al. 2004; (8) Berger et al. 2003a; (9) Soderberg et al. 2007; (10) Cenko et al. 2010; (11) Frail et al. 2006; (12) Rol et al. 2007; (13) Soderberg et al. 2006; (14) Chandra et al. 2008; (15) Gao 2009; (16) Rossi et al. 2011; (17) Cenko et al. 2011; (18) Chandra et al. 2010.

afterglow modeling studies on the same GRBs. In Table 6, for these early afterglow studies, we show the results from Panaitescu \& Kumar (2001a, 2001b, 2002) since they have the largest afterglow modeling compilations. It is important to note that in some cases, different groups determined significantly different values for the afterglow parameters (e.g., GRB 970508 Wijers \& Galama 1999; Chevalier \& Li 2000; Frail et al. 2000; Panaitescu \& Kumar 2002, GRB 000418 Berger et al. 2001; Panaitescu \& Kumar 2002).

\section{REFERENCES}

Achterberg, A., Gallant, Y. A., Kirk, J. G., \& Guthmann, A. W. 2001, MNRAS, 328,393

Antonelli, L. A., Testa, V., Romano, P., et al. 2006, A\&A, 456, 509

Barniol Duran, R. 2013, arXiv:1311.1216

Barniol Duran, R., \& Kumar, P. 2011, MNRAS, 417, 1584

Barthelmy, S. D., Barbier, L. M., Cummings, J. R., et al. 2005, SSRv, 120, 143 Beck, R. 2011, SSRv, 166, 215

Berger, E., Diercks, A., Frail, D. A., et al. 2001, ApJ, 556, 556 
Berger, E., Kulkarni, S. R., Pooley, G., et al. 2003a, Natur, 426, 154 Berger, E., Soderberg, A. M., Frail, D. A., \& Kulkarni, S. R. 2003b, ApJL, 587, L5

Bhattacharya, D. 2001, BASI, 29, 107

Björnsson, G., Gudmundsson, E. H., \& Jóhannesson, G. 2004, ApJL, 615, L77

Blandford, R. D., \& McKee, C. F. 1976, PhFl, 19, 1130

Burrows, D. N., Hill, J. E., Nousek, J. A., et al. 2005, SSRv, 120, 165

Butler, N. R., \& Kocevski, D. 2007, ApJ, 663, 407

Cenko, S. B., Frail, D. A., Harrison, F. A., et al. 2010, ApJ, 711, 641

Cenko, S. B., Frail, D. A., Harrison, F. A., et al. 2011, ApJ, 732, 29

Chandra, P., Cenko, S. B., Frail, D. A., et al. 2008, ApJ, 683, 924

Chandra, P., Frail, D. A., Fox, D., et al. 2010, ApJL, 712, L31

Chang, P., Spitkovsky, A., \& Arons, J. 2008, ApJ, 674, 378

Chevalier, R. A., \& Li, Z.-Y. 1999, ApJL, 520, L29

Chevalier, R. A., \& Li, Z.-Y. 2000, ApJ, 536, 195

Chincarini, G., Moretti, A., Romano, P., et al. 2007, ApJ, 671, 1903

Couch, S. M., Milosavljević, M., \& Nakar, E. 2008, ApJ, 688, 462

Covino, S., Campana, S., Conciatore, M. L., et al. 2010, A\&A, 521, A53

Curran, P. A., Evans, P. A., de Pasquale, M., Page, M. J., \& van der Horst, A. J. 2010, ApJL, 716, L135

Eichler, D., \& Waxman, E. 2005, ApJ, 627, 861

Evans, P. A., Beardmore, A. P., Page, K. L., et al. 2007, A\&A, 469, 379

Evans, P. A., Beardmore, A. P., Page, K. L., et al. 2009, MNRAS, 397, 1177

Fan, Y., \& Piran, T. 2006, MNRAS, 369, 197

Frail, D. A., Cameron, P. B., Kasliwal, M., et al. 2006, ApJL, 646, L99

Frail, D. A., Waxman, E., \& Kulkarni, S. R. 2000, ApJ, 537, 191

Gao, W.-H. 2009, ApJ, 697, 1044

Gehrels, N., Chincarini, G., Giommi, P., et al. 2004, ApJ, 611, 1005

Gehrels, N., Ramirez-Ruiz, E., \& Fox, D. B. 2009, ARA\&A, 47, 567

Goodman, J., \& MacFadyen, A. 2008, JFM, 604, 325

Gotz, D., \& Mereghetti, S. 2005, GCN, 3329, 1

Granot, J., Königl, A., \& Piran, T. 2006, MNRAS, 370, 1946

Granot, J., \& Sari, R. 2002, ApJ, 568, 820

Greiner, J., Krühler, T., Fynbo, J. P. U., et al. 2009, ApJ, 693, 1610

Gruzinov, A., \& Waxman, E. 1999, ApJ, 511, 852

Guidorzi, C., Kobayashi, S., Perley, D. A., et al. 2011, MNRAS, 417, 2124

Harrison, F. A., Yost, S. A., Sari, R., et al. 2001, ApJ, 559, 123

Inoue, T., Asano, K., \& Ioka, K. 2011, ApJ, 734, 77

Keshet, U., Katz, B., Spitkovsky, A., \& Waxman, E. 2009, ApJL, 693, L127

Krühler, T., Greiner, J., McBreen, S., et al. 2009, ApJ, 697, 758

Kumar, P., \& Barniol Duran, R. 2009, MNRAS, 400, L75

Kumar, P., \& Barniol Duran, R. 2010, MNRAS, 409, 226

Kumar, P., \& Panaitescu, A. 2000, ApJL, 541, L51

Lemoine, M. 2013, MNRAS, 428, 845

Lemoine, M., Li, Z., \& Wang, X.-Y. 2013, MNRAS, 435, 3009

Leventis, K., van Eerten, H. J., Meliani, Z., \& Wijers, R. A. M. J. 2012, MNRAS, 427, 1329

Li, Z.-Y., \& Chevalier, R. A. 2001, ApJ, 551, 940

Liang, E.-W., Racusin, J. L., Zhang, B., Zhang, B.-B., \& Burrows, D. N. 2008, ApJ, 675, 528

Liang, E.-W., Yi, S.-X., Zhang, J., et al. 2010, ApJ, 725, 2209

Martins, S. F., Fonseca, R. A., Silva, L. O., \& Mori, W. B. 2009, ApJL, 695, L189

Medvedev, M. V., Fiore, M., Fonseca, R. A., Silva, L. O., \& Mori, W. B. 2005, ApJL, 618, L75

Medvedev, M. V., \& Loeb, A. 1999, ApJ, 526, 697
Melandri, A., Kobayashi, S., Mundell, C. G., et al. 2010, ApJ, 723, 1331

Melandri, A., Mundell, C. G., Kobayashi, S., et al. 2008, ApJ, 686, 1209

Meszaros, P., \& Rees, M. J. 1993, ApJ, 405, 278

Meszaros, P., \& Rees, M. J. 1997, ApJ, 476, 232

Milosavljević, M., \& Nakar, E. 2006, ApJ, 651, 979

Milosavljevic, M., Nakar, E., \& Zhang, F. 2007, arXiv:0708.1588

Mizuno, Y., Pohl, M., Niemiec, J., et al. 2011, ApJ, 726, 62

Molinari, E., Vergani, S. D., Malesani, D., et al. 2007, A\&A, 469, L13

O'Brien, P. T., Willingale, R., Osborne, J., et al. 2006, ApJ, 647, 1213

Paczynski, B., \& Rhoads, J. E. 1993, ApJL, 418, L5

Panaitescu, A. 2005, MNRAS, 363, 1409

Panaitescu, A. 2007, MNRAS, 379, 331

Panaitescu, A., \& Kumar, P. 2000, ApJ, 543, 66

Panaitescu, A., \& Kumar, P. 2001a, ApJL, 560, L49

Panaitescu, A., \& Kumar, P. 2001b, ApJ, 554, 667

Panaitescu, A., \& Kumar, P. 2002, ApJ, 571, 779

Panaitescu, A., Mészáros, P., Burrows, D., et al. 2006, MNRAS, 369, 2059

Panaitescu, A., \& Vestrand, W. T. 2011, MNRAS, 414, 3537

Perley, D. A., Bloom, J. S., Klein, C. R., et al. 2009, MNRAS, 406, 2473

Perley, D. A., Li, W., Chornock, R., et al. 2008, ApJ, 688, 470

Perley, D. A., Morgan, A. N., Updike, A., et al. 2011, AJ, 141, 36

Piran, T. 2004, RvMP, 76, 1143

Piro, L. 2001, in Gamma-ray Bursts in the Afterglow Era, ed. E. Costa, F. Frontera, \& J. Hjorth (Berlin: Springer), 97

Rees, M. J., \& Meszaros, P. 1992, MNRAS, 258, 41P

Resmi, L., \& Bhattacharya, D. 2008, MNRAS, 388, 144

Robishaw, T., Quataert, E., \& Heiles, C. 2008, ApJ, 680, 981

Rol, E., van der Horst, A., Wiersema, K., et al. 2007, ApJ, 669, 1098

Rossi, A., Schulze, S., Klose, S., et al. 2011, A\&A, 529, A142

Sari, R., \& Piran, T. 1999a, ApJL, 517, L109

Sari, R., \& Piran, T. 1999b, ApJ, 520, 641

Sari, R., Piran, T., \& Narayan, R. 1998, ApJL, 497, L17

Schulze, S., Klose, S., Björnsson, G., et al. 2011, A\&A, 526, A23

Silva, L. O., Fonseca, R. A., Tonge, J. W., et al. 2003, ApJL, 596, L121

Sironi, L., \& Goodman, J. 2007, ApJ, 671, 1858

Sironi, L., \& Spitkovsky, A. 2011, ApJ, 726, 75

Sironi, L., Spitkovsky, A., \& Arons, J. 2013, ApJ, 771, 54

Soderberg, A. M., Berger, E., Kasliwal, M., et al. 2006, ApJ, 650, 261

Soderberg, A. M., Nakar, E., Cenko, S. B., et al. 2007, ApJ, 661, 982

Stratta, G., Pozanenko, A., Atteia, J.-L., et al. 2009, A\&A, 503, 783

Thompson, T. A., Quataert, E., \& Murray, N. 2009, MNRAS, 397, 1410

Ubertini, P., Lebrun, F., Di Cocco, G., et al. 2003, A\&A, 411, L131

Uehara, T., Uemura, M., Kawabata, K. S., et al. 2010, A\&A, 519, A56

Weibel, E. S. 1959, PhRvL, 2, 83

Wijers, R. A. M. J., \& Galama, T. J. 1999, ApJ, 523, 177

Winkler, C., Courvoisier, T. J.-L., Di Cocco, G., et al. 2003, A\&A, 411, L1

Xue, R.-R., Fan, Y.-Z., \& Wei, D.-M. 2009, A\&A, 498, 671

Yost, S. A., Frail, D. A., Harrison, F. A., et al. 2002, ApJ, 577, 155

Yost, S. A., Harrison, F. A., Sari, R., \& Frail, D. A. 2003, ApJ, 597, 459

Yuan, F., Schady, P., Racusin, J. L., et al. 2010, ApJ, 711, 870

Zhang, B. 2011, CRPhy, 12, 206

Zhang, B., Fan, Y. Z., Dyks, J., et al. 2006, ApJ, 642, 354

Zhang, B., Liang, E., Page, K. L., et al. 2007a, ApJ, 655, 989

Zhang, B.-B., Liang, E.-W., \& Zhang, B. 2007b, ApJ, 666, 1002

Zhang, W., MacFadyen, A., \& Wang, P. 2009, ApJL, 692, L40 